Check for updates

Cite this: Mater. Horiz., 2020, 7, 2276

Received 8th April 2020, Accepted 22nd June 2020

DOI: 10.1039/d0mh00586j

rsc.li/materials-horizons

\section{Advances in design engineering and merits of electron transporting layers in perovskite solar cells}

\author{
Han Pan, ${ }^{a}$ Xiaojuan Zhao, ${ }^{a}$ Xiu Gong, ${ }^{a}$ Hao Li, (D) a Najib Haji Ladi, ${ }^{b}$ Xiao Li Zhang, ${ }^{c}$ \\ Wenchao Huang, (D) def Shahzada Ahmad, (D) gh Liming Ding, (D) ' Yan Shen, (D) ${ }^{a}$ \\ Mingkui Wang (D) *a and Yongqing Fu (D) *bj
}

\begin{abstract}
The emerging photovoltaic technology, namely perovskite photovoltaics, follows a style to the blooming flower with superficial prosperity described by the ancient poet Yuxi Liu. Metal-halide perovskite has become one of the most promising photovoltaic materials for thin-film solar-cell applications, mainly due to its exellent visible light capture capability, large diffusion coefficients and long diffusion lengths of charge carriers (both electrons and holes). The reported power conversion efficiency of laboratory produced perovskite solar cells (PSCs) has exceeded 25\%. However, this value was only achieved for devices of less than $0.1 \mathrm{~cm}^{2}$ dimensions, and decreases significantly with the increase in area (for example, down to $11.6 \%$ for a device area of $\sim 800 \mathrm{~cm}^{2}$, showing the issues with the scalability). In PSCs, the electron transport layer (ETL) has to perform two essential functions, which are related: (i) to collect and transfer charges after the injection of electrons from the perovskite light harvester; and (ii) to block the backflow of holes, thus realizing effective charge separation and suppressing charge carrier recombination. However, there are several critical issues associated with the ETLs which limit the further development of the PSCs. For example, the experimentally obtained open-circuit voltages in PSCs are typically much lower compared to the theoretical voltage limit with their optical bandgaps between 1.59 and $1.63 \mathrm{eV}$. This is mainly attributed to inefficient charge transport in the PSCs due to inhomogeneous charge accumulation and serious interfacial recombination at the interfaces between the ETL and the perovskite active layer. We scan recent developments of ETLs in PSCs, and discuss design methodologies, mechanisms, processing and characterization of new types of ETLs. High power conversion efficiency with large open-circuit voltages/photo-currents could be achieved through designing materials and microstructures for high-quality ETLs with a high electron mobility, high electrical conductivity and pinhole-free film morphology. It is crucial to balance the charge diffusion processes in the respective charge collective layers of PSCs as the interfaces between the ETLs and perovskites along with their interfacial structures play key roles in achieving new solid-state mesoscopic solar cells optimized open circuit voltages and output photocurrents.
\end{abstract}

\footnotetext{
${ }^{a}$ Wuhan National Laboratory for Optoelectronics, School of Optoelectronic Science and Engineering, Huazhong University of Science and Technology, Wuhan 430074, China.E-mail: mingkui.wang@hust.edu.cn

${ }^{b}$ China-EU Institute for Clean and Renewable Energy, Huazhong University of Science and Technology, Luoyu Road 1037, Wuhan 430074, P. R. China

${ }^{c}$ State Centre for International Cooperation on Designer Low-Carbon \& Environmental Materials, School of Materials Science and Engineering, Zhengzhou University, Zhengzhou 450001, P. R. China

${ }^{d}$ Department of Materials Science and Engineering, Monash University, Clayton, Victoria 3800, Australia

${ }^{e}$ State Key Laboratory of Advanced Technology for Materials Synthesis and Processing, Wuhan University of Technology, Wuhan 430070, P. R. China

${ }^{f}$ Foshan Xianhu Laboratory of the Advanced Energy Science and Technology Guangdong Laboratory, Xianhu Hydrogen Valley, Foshan, 528216, P. R. China

${ }^{g}$ BCMaterials-Basque Center for Materials, Applications \& Nanostructures, BC Materials, Leioa, 48940 Bilbao, Spain

${ }^{h}$ IKERBASQUE, Basque Foundation for Science, Bilbao, 48013, Spain

${ }^{i}$ National Center for Nanoscience and Technology, Beijing 100190, P. R. China

${ }^{j}$ Faculty of Engineering and Environment, Northumbria University, Newcastle Upon Tyne, NE1 8ST, UK. E-mail: richard.fu@northumbria.ac.uk
} 


\section{Introduction}

Organic-inorganic hybrid lead halide perovskite solar cells (PSCs) have received extensive attention recently, mainly because the output power conversion efficiency (PCE) has been increased rapidly from $3.9 \%$ in 2009 to $25.2 \%$ in $2019 .^{1,2}$ These organic-inorganic hybrid lead perovskites have a general formula of $\mathrm{ABX}_{3}$ with an octahedral structure (as illustrated in Fig. 1a), where the component A, generally forming the cube unit cell, represents $\mathrm{CH}_{3} \mathrm{NH}_{3}{ }^{+}$(MA), $\mathrm{HC}\left(\mathrm{NH}_{2}\right)_{2}{ }^{+}$(FA) or $\mathrm{Cs}^{+}$ cation; the component $\mathrm{B}$, which is located in the centre of the cube unit cell, represents $\mathrm{Pb}^{2+}$ or $\mathrm{Sn}^{2+}$; and the component $\mathrm{X}$, generally located at surface centres of the cube unit cell of $\mathrm{A}$, represents halogen ions such as $\mathrm{Cl}^{-}, \mathrm{Br}^{-}$or $\mathrm{I}^{-} .4,4$

Depending on the light incidence, PSCs can be broadly divided into either $n-i-p$ or $p-i-n$ architectures, where $n-$ and p- refer to n-type and p-type charge carrier transporting materials, respectively, and i refers to the perovskite optical absorption layer. This definition is also based on the fact whether the electron transporting layer (ETL) or the hole-transporting layer (HTL) is designed to contact with the transparent conductive substrates. For example, in a conventional planar PSC, an ETL can be deposited on the transparent-conductive oxide (TCO) layer such as fluorine-doped tin oxide (FTO) or indium tin oxide (ITO) which will function as the cathode, and then a perovskite layer is prepared on top of the ETL using spin-coating or vacuum evaporation processes. This is then followed by the successive deposition of an HTL and a metal electrode, such as Au or Ag, to form an $n-\mathrm{i}-\mathrm{p}$ structured device (Fig. 1b). ${ }^{5-9}$ In contrast, in an inverted PSC design, the positions of the ETL and HTL are simply exchanged (see Fig. 1c). ${ }^{10-14}$

Compared to the planar n-i-p structured PSCs, the mesoporous PSCs utilize a compact $\mathrm{TiO}_{2}$ layer formed on FTO or ITO substrates and then an additional mesoporous $\mathrm{TiO}_{2}$ layer is deposited as both the ETL and structural scaffold. ${ }^{15-17}$ Afterwards, the perovskite layer is deposited to form the absorber layer followed by fabrication of the HTL $\left(2,2^{\prime}, 7,7^{\prime}\right.$-tetrakis-( $N, N$-di- $p$-methoxyphenylamine)-9,9'-spiro-bifluorene (spiro-OMeTAD) or poly[bis(4phenyl)(2,4,6-trimethyl-phenyl)amine] (PTAA)) and metal electrode (Fig. 1d). A special feature for the mesoporous structured PSCs is a)

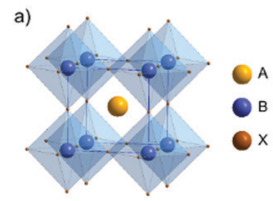

c)

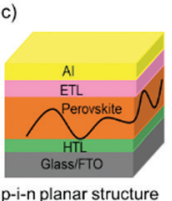

d)

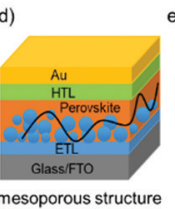

b)

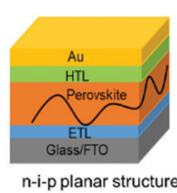

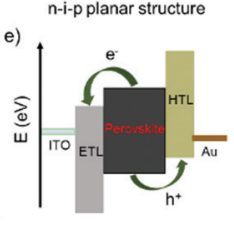

Fig. 1 (a) Illustration of a crystalline structure of perovskite $A B X_{3}, A$ : $M A^{+}$ or $\mathrm{FA}^{+}$cation, $\mathrm{B}: \mathrm{Pb}^{2+}$ cation, $\mathrm{X}: \mathrm{Cl}^{-}, \mathrm{Br}^{-}$or $\mathrm{I}^{-}$anion. Typical perovskite solar cells with (b) $n-i-p$ structure, (c) $p-i-n$ structure, and (d) mesoporous structure. (e) Energy level scheme for the transporting direction of electron and hole carriers in $n-i-p$ perovskite solar cells.

that the mesoscopic transport layer offers large contact areas and interfacial roughness for perovskite layer deposition. Consequently, different outcomes of photocurrent hysteresis and output photovoltage for the planar and mesoporous structured PSC devices can be realized.

One of the key figures of merits for the PSC technology is that the devices can be fabricated using low-temperature solution-processed methods such as spin-coating and screenprinting, which can be easily mass-produced in industry. Upon being irradiated by sunlight, electron-hole pairs (i.e., excitons) are generated in the light-harvesting perovskite layer. After separation of the electron-hole pairs (induced by thermal energy due to a low exciton binding energy), the photogenerated electrons are transported to the conduction band of the ETL, and the holes are transported to the highest occupied molecular orbital (HOMO) energy level of the HTL, and are then collected by the cathode and anode, respectively (in Fig. 1e). ${ }^{18,19}$

\subsection{Brief development of perovskite solar cells}

The organic-inorganic metal halide perovskite materials ( $\left.\mathrm{MAPbI}_{3}\right)$ were firstly utilized as both a sensitizer and light absorber in solar cells by Miyasaka et al. in $2009,{ }^{20}$ though with a low photoelectric PCE of 3.8\%. In 2011, Park et al. utilized $\mathrm{MAPbI}_{3}$ perovskite quantum dots (size of $2-3 \mathrm{~nm}$ ) as the pigment in sensitized solar cells, achieving a high PCE of $6.5 \%{ }^{21}$ They reported that the light absorption coefficient of the $\mathrm{MAPbI}_{3}$ nanocrystals is one order of magnitude higher than that of the conventional dye, thus $\mathrm{MAPbI}_{3}$ could be effectively used in solid-state sensitized solar cells. In this type of solar cells, a much thinner $\mathrm{TiO}_{2}$ layer $(\sim 2 \mu \mathrm{m})$ is widely used. More importantly, a new solid-state mesoscopic solar cell has been developed which can prevent liquid electrolytes from dissolving perovskite, and thus improve its stability. ${ }^{22}$ In 2012, Park et al. reported $\sim 10 \%$ efficiency for perovskite-based solid-state solar cells by adopting small molecular spiro-OMeTAD as the HTL to replace the iodide-based liquid electrolyte. ${ }^{23}$ Furthermore, Snaith et al. reported that utilization of an $\mathrm{Al}_{2} \mathrm{O}_{3}$ mesoporous layer as the scaffold for the PSCs could achieve much faster electron transport with the perovskite layer, if compared with that using the n-type $\mathrm{TiO}_{2}$ with the perovskite layer. ${ }^{24}$ Later, a simplified planar structured PSC was successfully developed with the help of the bipolar carrier transport property of perovskite compounds. ${ }^{25}$

Fig. 2 summarizes the key development milestones for the PSCs. For the $\mathrm{MAPbI}_{3}$ perovskite film, a sequential deposition via a two-step dip coating process was developed as one of the effective methods to achieve efficient solution-processed photovoltaic cells with a good reproducibility. ${ }^{26}$ Park et al. found that the deposition procedure (e.g., one-step ${ }^{27}$ and two-step coating methods ${ }^{28}$ ) of the perovskite layer changes not only the electron lifetime but also the film morphology, which plays a critical role in device performance. Afterwards, the PCE for small-area perovskite devices $\left(\sim 0.1 \mathrm{~cm}^{2}\right)$ increased rapidly to above $25 \%$ within 10 years. However, when the device size increases up to $\sim 800 \mathrm{~cm}^{2}$, the PCE of PSCs is decreased to $11.6 \% .{ }^{29}$ which is 


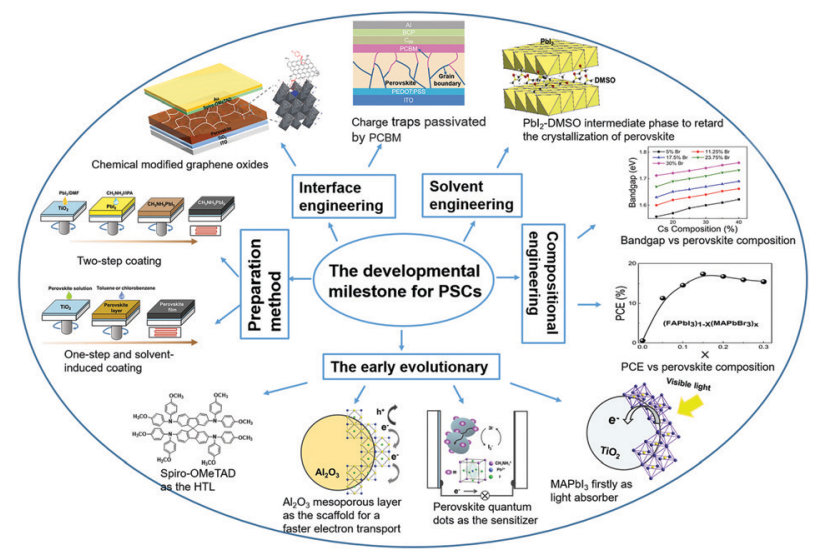

Fig. 2 Illustration of the developmental milestones for perovskite solar cells, modified from ref. 24, 29, 38, 42 and 44.

much lower than that of silicon-based solar cells $(\sim 26.7 \%) .^{30,31}$ This indicates that the scalability is a crucial issue for the successful commercialization of the PSCs.

In order to enhance the PSCs' stability in operational conditions and reduction in production costs, various key factors controlling the charge carrier collection within the charge transport layers and their contacts to the absorber layers have been extensively investigated. ${ }^{19}$ In order to achieve their high efficiencies, the PSCs inevitably require an optimized carrier transport pathway that leverages all of the device layers and their corresponding interfaces. In this regard, interface engineering ${ }^{32-34}$ has become an effective strategy to tune the optoelectronic properties of the solar cell devices. So far, different methods, such as Lewis base passivation ${ }^{35}$ and fullerene passivation, ${ }^{36}$ have been demonstrated to significantly improve solar cell efficiency. For example, the $\mathrm{PbI}_{2}$ (Lewis acid) tends to have strong interactions with those sulfur, oxygen, or nitrogen contained polar aprotic solvents (Lewis base), ${ }^{37}$ including dimethyl sulfoxide (DMSO), ${ }^{38} \mathrm{~N}, \mathrm{~N}$-dimethylformamide (DMF) ${ }^{39}$ and $N$-methylpyrrolidone. ${ }^{40}$

Although $\mathrm{MAPbI}_{3}$ has been widely used as an active layer in PSCs, it has some disadvantages such as unstable crystalline phases under high humidity and within the solvents. These problems could be solved by using the solvent engineering methods. For instance, a mixed solvent of $\gamma$-butyrolactone and DMSO leads to extremely uniform and dense perovskite layers via a $\mathrm{CH}_{3} \mathrm{NH}_{3} \mathrm{I}-\mathrm{PbI}_{2}-\mathrm{DMSO}$ intermediate phase, and thus enables the fabrication of solar cells with a remarkably increased PCE of $16.2 \%$ and without photocurrent hysteresis. ${ }^{41} \mathrm{An}$ absorber based on $\mathrm{Cs}_{0.25} \mathrm{FA}_{0.75} \mathrm{PbI}_{2.40} \mathrm{Br}_{0.60}$ was reported to have a PCE of $17.5 \%$ with both good stability and reproducibility. ${ }^{42,43}$ Seok et al. applied the compositional engineering methodology to develop a $\left(\mathrm{FAPbI}_{3}\right)_{0.85}\left(\mathrm{MAPbBr}_{3}\right)_{0.15}$ perovskite solar cell and achieved a high PCE of $19.0 \%$, which would bring balance between electron and hole transports within the perovskite layer. ${ }^{44}$

\subsection{Charge transport in perovskite solar cells}

Fig. 3a presents a scheme of a heterojunction formed among the ETL/perovskite layer/HTL, and Fig. 3b shows the representative

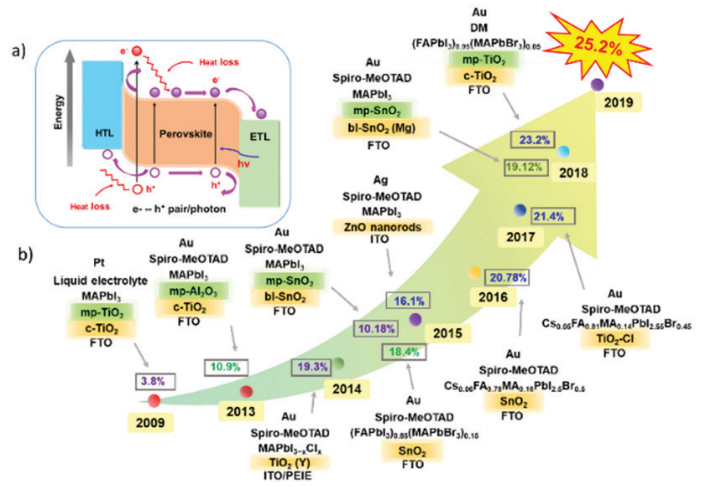

Fig. 3 (a) Schematic diagram and energy band of interfaces among the electron transport layer (ETL)/perovskite/hole transport layer (HTL). (b) The representative progress of ETLs in the $n-i-p$ architecture.

development progress of ETLs in the $\mathrm{n}-\mathrm{i}-\mathrm{p}$ architecture. The ETL (usually highly doped $\mathrm{n}$-type metal oxides, donated as $\mathrm{n}^{+}$) must possess a high electron concentration to conduct the electrons to the cathode with a small voltage drop. Under a given bias voltage across the device terminals, the electrical current through the ETL/ perovskite and perovskite/HTL contact interfaces must be the same as those of the rest of the device. Therefore, the competition between charge carrier recombination and charge transport is critical for the PSCs which have a typical total thickness of about sub-micrometre. It becomes critical how to further reduce charge carrier recombination at interfaces between ETL/perovskite, and choose the best contact electrode for the PSC devices.

Highly efficient PSCs generally possess a common feature of two interfaces, e.g., ETL/perovskite interface and HTL/perovskite heterojunction interface, both of which enable efficient charge separation and collection. Fig. 4 illustrates various charge carrier kinetics at these two interfaces, including charge transfer, charge carrier collection and charge carrier recombination. The desirable processes include: (1) photo-excitation in the perovskite; (2) electron transfer to the ETL; and (3) hole transfer to the HTM (or, equivalently, electron transfer from the HTM to the perovskite). The undesirable processes include: (4) bulk recombination of photo-generated species; (5) back charge transfer at the interfaces of $\mathrm{TiO}_{2}$, and (6) charge transfer at the HTM with the perovskite; and (7) charge transfer between $\mathrm{TiO}_{2}$ and the HTM

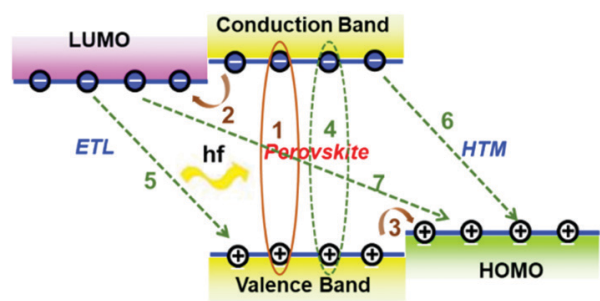

Fig. 4 Diagram for charge transfer, collection and recombination of planar PSCs with a structure of ITO/ETL/MAPb/ ${ }_{3} / \mathrm{HTM} / \mathrm{Au}$. Photon exciton (1), charge transfer (2 and 3), collection and recombination $(4,5,6,7)$ steps take place at the interfaces. The thick green and thin red arrows respectively indicate the processes desirable for energy conversion and those associated with losses. hf, photon energy. 
(this may occur if perovskite is absent in some areas, for example, when nanoparticles or voids are present). For achieving highperformance PSCs, processes (4)-(7) must be performed on much slower timescales than charge generation and extraction processes (1)-(3). The interface recombination (non-radiative process) occurs at the ETL/perovskite or perovskite/HTL interface; it is also often called the free-carrier recombination (bimolecular recombination) and can be experimentally verified by investigating the slope of $V_{\mathrm{OC}}$ versus light intensity. For efficient planar PSC devices, a slope of lower than $1 \mathrm{kT} / q$ (being $0.0256 \mathrm{dec}^{-1}$ at room temperature) has been frequently observed. ${ }^{45}$

The ETLs in PSCs play important roles in collecting and transferring charge carriers after the injection of electrons from a perovskite active layer, more importantly, realizing effective charge separation and suppressing charge carrier recombination. An inefficient charge transport could cause inhomogeneous charge accumulation and serious interfacial recombination at the ETL/perovskite interface. Therefore, it is highly desirable to design and fabricate ETLs with a high quality in order to guarantee an efficient charge transport in the layer and thus effective photovoltaic performance of devices.

Indeed, each layer (its crystallinity and quality) and the interfacial properties between different layers are critical to achieving highly efficient PSCs. ${ }^{46-49}$ This can be significantly documented with several critical progresses on perovskite devices and the relative topics. ${ }^{20,23}$ This article covers the cutting-edge research areas, recent development, critical issues and future directions of ETL materials for PSC applications, with a focus on the following major topics: the functions and requirements of the ETL, and the techniques utilized in the characterization of the ETL. Herein, we briefly discuss the recent advances in design methodology, mechanism and characterization of ETLs. We also analyse ETL material properties including their composition and morphology. We believe readers can also obtain interesting and informative knowledge from different sources in this community. ${ }^{50-53}$

\section{Functions and requirements of electron transport layers}

Recently, there have been extensive studies to search for effective ETLs to be used in planar PSCs to further increase charge carrier transport. The main reason is that the $V_{\text {OC }}$ values (in the range of $\sim 1.0-1.2 \mathrm{~V}$ ) of most PSCs made from organicinorganic halide lead perovskites are not large enough compared to the typical optical bandgap (between 1.59 and $1.63 \mathrm{eV}$ ) of the light absorber. The open circuit losses in the PSCs are mainly due to the recombination occurring in the perovskite bulk layer, and at either the perovskite/transport layer interfaces or the transport layers. Furthermore, the ETL plays critical roles in increasing the power conversion efficiency and lifetime of the devices. Usually, such charge carrier contact layers are composed of metal oxide nanocrystals, such as $\mathrm{ZnO}, \mathrm{TiO}_{x}$, or doped metal oxides, such as Cl-doped $\mathrm{TiO}_{2}$ (in Fig. $3 \mathrm{~b}$ ). ${ }^{54}$ A reliable method for producing large-area ETLs is one of the pre-requisites for successful commercialization of the PSC technology. Still, we have to mention the importance of ETL in n-i-p structured PSCs has been long overlooked not only in the preparation procedures of devices but also augments of the performance, mainly due to its thin thickness property (less than $100 \mathrm{~nm}$ ) and difficulty of controlling the morphology. This is also happened for the HTL in p-i-n structured PSC devices.

The mechanism for charge transfer through metal oxide semiconductor nanocrystalline particles and surface charge carrier recombination is illustrated in Fig. 5a, along with a charge injection-accumulation-diffusion model for charge transfer dynamics of those semiconducting nanocrystals, as shown in Fig. 5b. It is clear that defect states in these nanocrystals play a critical role in the charge transport process (capture and release processes). Due to the influence of trapdetrap from defect states, the conductivity of nanocrystals is generally lower than that of the crystalline film. Therefore, electron transport and conductivity mechanisms in metal oxide nanocrystals will significantly influence the efficiency of energy photo conversion in such types of photovoltaic devices.

A nanocrystalline metal oxide film can be modelled as a network of junctions formed from the connected nanoparticles. Fig. 5c shows a cross-section scanning electron microscope (SEM) image of a typical planar $\mathrm{ITO} / \mathrm{SnO}_{2} / \mathrm{MAPbI}_{3} /$ spiro-OMeTAD $/ \mathrm{Au}$ PSC. The charge transport layer thickness is $\sim 40 \mathrm{~nm}^{55}$ A fluctuation-induced tunnelling conductivity (FITC) mechanism has been proposed for the charge transport in nanoporous metal oxides such as $\mathrm{TiO}_{2}$ and $\mathrm{SnO}_{2}$, and the charge transport in these films is strongly dependent on the properties of the junctions linking these nanoparticles. ${ }^{56}$ The FITC model describes contact junctions of these nanoparticles as effective potential energy barriers for transferring electrons, owing to the intrinsic disorder a)

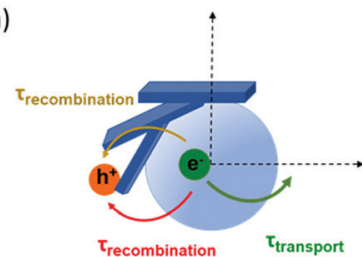

c)

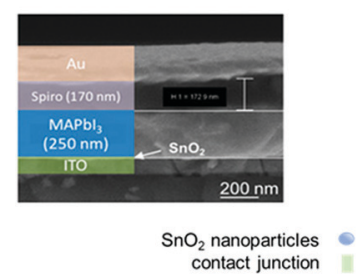

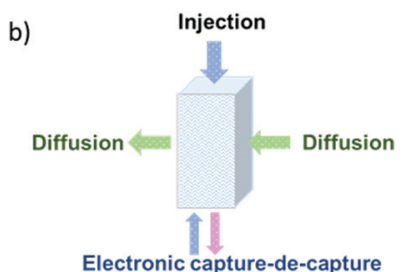

d)

e)

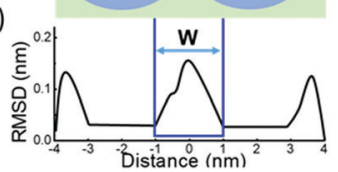

Fig. 5 (a) Schematic illustration of the electronic accumulation-diffusion model on a nanocrystalline particle. (b) Effect of defect states in nanocrystalline materials on charge transport. (c) The cross-sectional scanning electron microscope (SEM) image of a planar $1 \mathrm{TO} / \mathrm{SnO}_{2} /$ perovskite/ spiro-OMeTAD/Au device. (d) Contact junction (green) of nanocrystalline $\mathrm{SnO}_{2}$ nanoparticles (blue). Molecular dynamics simulation results of two $\sim 4 \mathrm{~nm}$ particles. (e) Average atomic root-mean-squared deviation (RMSD) relative to $\mathrm{SnO}_{2}$ particles in figure d. (c) Reproduced with permission from ref. 55. Copyright 2017 American Chemical Society. 
of the semiconductor material between the nanocrystalline particles (Fig. 5d and e).

For photovoltaic device applications, the mobility of electrons in the ETL must be high enough to increase the electronic conductivity. Electron mobility $\left(\mu_{\mathrm{e}}\right)$ can be expressed using eqn (1):

$$
\mu_{n}=\frac{e \tau}{m_{\mathrm{e}}^{*}}
$$

where $m_{\mathrm{e}}{ }^{*}$ is the electron effective mass, $\tau$ is the average free time and $e$ is the elementary charge. The electron mobility is proportional to the electron free time, being correlated with the material properties and the mechanisms of carrier scattering. ${ }^{57}$ Electric conduction of semiconductors is directly proportional to the number of free electrons in the conduction band, e.g.,

$$
\sigma=n D E
$$

where $\sigma$ is the electrical conductivity, $n$ is the concentration of free electrons based on the Boltzmann relation, $D$ is the diffusion coefficient of electrons, and $E$ is the electric field. The relationship between electron mobility $(\mu)$ and diffusion coefficient $(D)$ can be defined by the Einstein equation. ${ }^{58}$ An increase of either charge carriers $(n)$ or mobility $\left(\mu_{n}\right)$ can increase the electrical conductivity of the ETLs. One can roughly estimate the electron conductivity and mobility required for the ETL in efficient PSCs. The electrical conductivity could be quantified as $\sigma=\left(d \times J_{\mathrm{SC}}\right) / V$, with the voltage drop $(V)$ equal or less than the thermal voltage $V_{\mathrm{T}}{ }^{146}$ Taking a theoretical value $J_{\mathrm{SC}}$ of $25 \mathrm{~mA} \mathrm{~cm} \mathrm{~cm}^{-2}$ for the PSC with a bandgap of $1.6 \mathrm{eV}^{147}$ and the thickness of $40 \mathrm{~nm}(d)$ for the ETL, the calculated $\sigma$ should be larger than $4 \times 10^{-6} \mathrm{~S} \mathrm{~cm}^{-1}$. Furthermore, the electron mobility can be estimated according to $\sigma=q \mu_{n} N_{\mathrm{D}}$, where $N_{\mathrm{D}}$ is the doping density of the donor and $q$ is the elementary charge. Based on the literature, the charge $N_{\text {D }}$ is estimated to be $\sim 1.0 \times 10^{18} \mathrm{~cm}^{-3}$ for most of the efficient solar cells, ${ }^{148}$ and therefore, the electron mobility $\mu_{n}$ can be estimated to be higher than $\sim 2.5 \times 10^{-5} \mathrm{~cm}^{2} \mathrm{~V}^{-1} \mathrm{~s}^{-1}$.

High-quality ETLs should have the following properties: (1) a suitable lowest unoccupied molecular orbital (LUMO) energy level to match with the conduction band energy of perovskite materials, together with good capabilities for electron injection and transportation at the interfaces; (2) high electron mobility $\left(>2.5 \times 10^{-5} \mathrm{~cm}^{2} \mathrm{~V}^{-1} \mathrm{~s}^{-1}\right)$, high electrical conductivity $\left(>4 \times 10^{-6} \mathrm{~S} \mathrm{~cm}^{-1}\right)$ and photochemical stability under ultraviolet light; and (3) conformal, uniform and pinhole-free film morphology with a low root-mean-square roughness. ${ }^{59}$ It should be noted that the above criteria for the parameters are for the optimised and highly efficient perovskite photovoltaic devices with low series resistance and high shutting resistance.

A suitable ETL should be also optically transparent to guarantee maximum light absorption by the perovskite layer in the $n-i-p$ PSC. This hinders the choice of suitable materials. For example, some organic materials might have problems with good transparency. The most popular inorganic ETLs are transition metal oxides such as $\mathrm{TiO}_{2},{ }^{60} \mathrm{ZnO},{ }^{46}$ and $\mathrm{SnO}_{2} \cdot{ }^{61}$
Although the PSC devices using a $\mathrm{TiO}_{2}$ ETL have achieved a relatively high efficiency (e.g., above $20 \%$ ), this metal oxide exhibits some drawbacks such as low electronic conductivity and charge transport. ${ }^{62,63}$ Hence, $\mathrm{ZnO}$ and $\mathrm{SnO}_{2}$, well known for their higher electron mobility (bulk mobility: $205-300 \mathrm{~cm}^{2}$ $\mathrm{V}^{-1} \mathrm{~s}^{-164}$ and $240 \mathrm{~cm}^{2} \mathrm{~V}^{-1} \mathrm{~s}^{-1},{ }^{63}$ respectively) than that of $\mathrm{TiO}_{2}$ (bulk mobility: $1 \mathrm{~cm}^{2} \mathrm{~V}^{-1} \mathrm{~s}^{-163}$ ), have emerged as promising ETLs. Last but not least, the stability of the ETL in the PSC devices under operation conditions is also critical for the commercialization of this technology. During the operation of PSCs, ETLs should effectively transport electrons from light absorption layers and prevent hole migration in the direction of electron migration.

\section{Characterization techniques utilized for the ETL in perovskite solar cells}

\subsection{Ultrafast spectroscopy characterization}

There are several methods available to characterize the charge carrier collection dynamics related to the ETLs such as the electron transport in this layer and interfacial charge carrier recombination at the ETL/perovskite junction. Nanosecond transient absorption spectroscopy (ns-TAS) has been used in order to understand charge injection at the ETL/perovskite interface. For example, photobleaching (PB) negative and photoabsorption (PA) positive peaks of the $\mathrm{MAPbI}_{3-x} \mathrm{Cl}_{x}$ based PSCs are usually observed at around 760 and 500-600 nm, respectively. The PB negative peak located at $760 \mathrm{~nm}$ can be linked to the band gap or exciton transition of the $\mathrm{MAPbI}_{3-x} \mathrm{Cl}_{x}$ film, whereas the PA positive peak at about $500-600 \mathrm{~nm}$ is attributed to the absorption of transient species. ${ }^{45,65}$ When the $\mathrm{MAPbI}_{3-x} \mathrm{Cl}_{x}$ was coated on an ETL substrate, an obvious decrease of the peak intensity (i.e., bleaching) was observed at about $760 \mathrm{~nm} .^{33}$ The kinetic decay of photo-bleaching features can be described using a single exponential function, which has a time constant $\tau_{\text {TAS }}$ being related to the excited-stated decay in the perovskite layer. This phenomenon well supports the result obtained from the steady-state PL quenching experiments, which confirms that the ETL contributes to the exciton transition or charge extraction process from the $\mathrm{MAPbI}_{3-x} \mathrm{Cl}_{x}$ film. ${ }^{66}$

Electron transport in the nano-crystalline metal oxide ETL can also been characterized using intensity modulated photocurrent spectroscopy (IMPS). ${ }^{67}$ Using small sinusoidal modulation optical signals, the IMPS could help analysis of transient photoelectric current and transient photovoltage response curves, as well as the characteristic parameters related to carrier transport dynamics such as electron diffusion coefficient and electron lifetime. ${ }^{68}$ This enables the study of electronic transport under steady state conditions for the photocurrent flow and occupation of electronic states.

\subsection{Electronic impedance spectroscopy characterization}

Electronic impedance spectroscopy (EIS) is a powerful tool to characterize the electronic and electrical processes in the PSCs, 
including charge extraction, diffusion and recombination in a thin layer or in the whole device. ${ }^{69}$ As an example, typical impedance spectra of a PSC using the device structure of FTO/ compact $\mathrm{TiO}_{2} /$ mesoporous $\mathrm{TiO}_{2}\left(\mathrm{MAPbI}_{3}\right)$ /spiro-OMeTAD/Au at different bias are shown in Fig. 6 .

In this measurement, the injected electrons from the FTO/ $\mathrm{TiO}_{2}$ compact contact are transported through mesoporous $\mathrm{TiO}_{2}$, and then diffuse within the $\mathrm{CH}_{3} \mathrm{NH}_{3} \mathrm{PbI}_{3}$ and spiroOMeTAD, eventually reaching the metal counter electrode (Fig. 7a). Frequency analysis showed three distinct frequency ranges (e.g., a high-frequency one at hundreds $\mathrm{kHz}$, a mediumfrequency one at hundreds $\mathrm{Hz}$, and a low-frequency one less than $\mathrm{Hz}$ ). As shown in Fig. 7b, the first arc feature in the high frequency of the Nyquist plot is assigned to the spiro-OMeTAD/ $\mathrm{Au}$ electrode interface and/or ETL/FTO interface (usually for the bias $>-0.85 \mathrm{~V}$ ). It should be noted that this arc would disappear if the interface is Ohmic contact, thus the presence of an arc indicates the formation of a Schottky junction at this interface. The second arc in the intermediate frequency range is assigned to the interfacial recombination at the ETL/perovskite interface and perovskite/HTL interface, which usually exponentially increases as the bias is decreased. These processes on both interfaces are sometimes difficult to be distinguished due to their similarity. In this frequency region, a Warburg impedance feature might show up which is linked to hole transport (Fig. 6a and b). Instead, a linear Warburg impedance feature appears in the middle frequency range when the bias is decreased from -0.75 to $-0.5 \mathrm{~V}$. (Fig. $6 \mathrm{c}$ and d). This particular feature arises from an increase in the electron resistance of the (mesoscopic) $\mathrm{TiO}_{2}$ thin film due to the decrease of electron concentration upon decreasing the forward bias. The gradual appearance of charge diffusion in semiconducting materials is

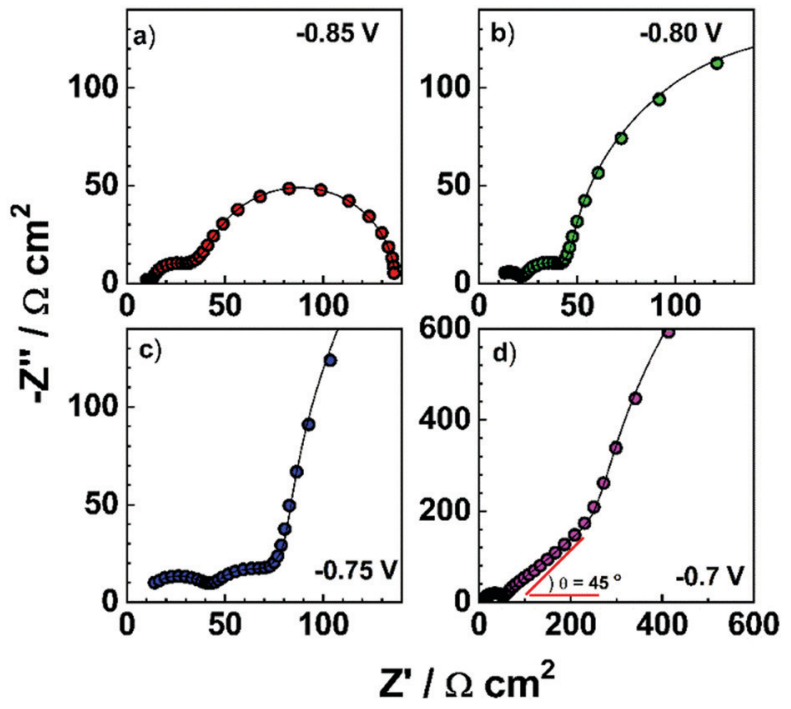

Fig. 6 Impedance spectra of a PSC with $\mathrm{FTO} /$ compact $\mathrm{TiO}_{2} /$ mesoporous $\mathrm{TiO}_{2}\left(\mathrm{MAPbl}_{3}\right)$ /spiro-OMeTAD/Au structure using mesoporous $\mathrm{TiO}_{2}$ ( $20 \mathrm{~nm}$ diameter, $800 \mathrm{~nm}$ thickness) film as a photo-anode filled with $\mathrm{MAPbl}_{3}$, obtained under dark conditions with different bias: (a-d) Nyquist plots $(-0.85,-0.8,-0.75$, and $-0.7 \mathrm{~V}$, respectively).

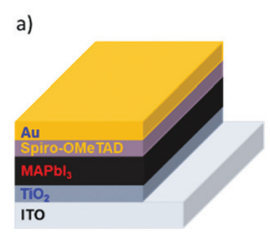

c)

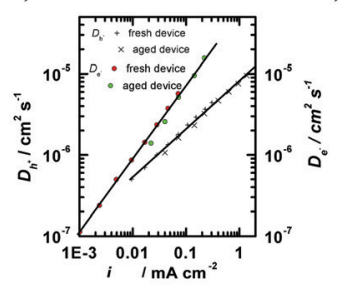

d)
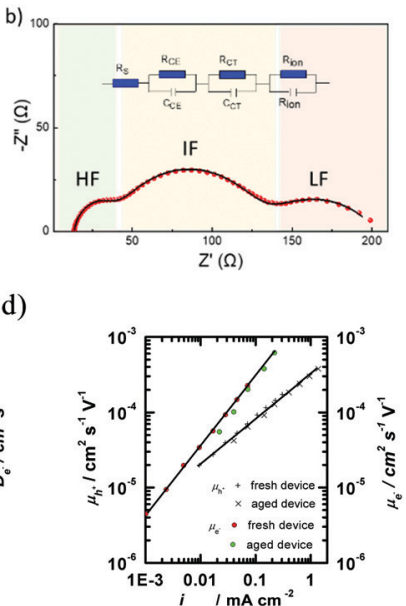

Fig. 7 (a) A PSC using a device structure of $1 \mathrm{TO} / \mathrm{TiO}_{2} / \mathrm{MAPbl}_{3} /$ spiroOMeTAD/Au. (b) Typical impedance spectroscopy (Nyquist plot) of this device and the corresponding equivalent circuit, in which HF indicates high frequency, IF indicates intermediate frequency, and LF indicates low frequency. ( $c$ and d) The diffusion coefficient and the mobility of holes (left coordinate) and electrons (right coordinate).

correlated with the semi-conductive properties of both the HTL and ETL used in the PSCs. This is a typical behaviour for semiconducting materials when the electronic Fermi level is far from the conduction or valence band energy. ${ }^{70}$ In this case, the charge carrier transport resistance can be used to obtain the electron/hole diffusion coefficient and mobility based on the three-channel transmission line impedance model (Fig. 7c and d). ${ }^{71}$ The third arc observed at the low frequency range corresponds to the charge transport in the perovskite layer, such as ion migration. It should be noted that a typical impedance spectrum in Fig. 7b is usually observed in the PSCs using a mesoporous charge transport layer. The features of charge transport in selective layers for the planar PSCs, in which very thin ETLs are used (i.e., low electronic resistance), become invisible by suitably tuning the bias during characterization using impedance spectroscopy.

The properties of electron transport in the ETL and hole transport in the HTL can be obtained by fitting the impedance spectroscopic data of $\mathrm{ITO} / \mathrm{TiO}_{2} / \mathrm{MAPbI}_{3} /$ spiro-OMeTAD/Au devices. For example, using EIS, we have estimated the hole mobility of a spiro-OMeTAD layer to be about $6 \times 10^{-4} \mathrm{~cm}^{2} \mathrm{~V}^{-1} \mathrm{~s}^{-1}$, higher than the electron mobility of $\mathrm{TiO}_{2}\left(\sim 8 \times 10^{-5} \mathrm{~cm}^{2} \mathrm{~V}^{-1} \mathrm{~s}^{-1}\right){ }^{72,73}$ Snaith et al. pointed out that the hole mobility of spiro-OMeTAD is higher than the electron mobility of $\mathrm{TiO}_{2}$ through testing the conductivities of the in-plane 'hole-only' and 'electron-only' devices based on the $\mathrm{TiO}_{2}$ and the spiro-OMeTAD, respectively. The hole conductivity is approximately three times higher than the electron conductivity, with the hole and electron mobilities being $3 \times 10^{-4} \mathrm{~cm}^{2} \mathrm{~V}^{-1} \mathrm{~s}^{-1}$ and $0.8 \times 10^{-4} \mathrm{~cm}^{2} \mathrm{~V}^{-1} \mathrm{~s}^{-1}$ for spiroOMeTAD and $\mathrm{TiO}_{2}$, respectively. ${ }^{74}$ The effective diffusion coefficient of electrons for the $\mathrm{TiO}_{2}$ nanocrystal decreases with the illumination intensities. In the meantime, the diffusion length is shorter than the film thickness. The results show that a reduction of electron transport resistance (for example, by reducing the film thickness, or by increasing the electronic mobility 
and/or free number of electrons) can significantly improve the PSCs' performance. ${ }^{75}$ This is realized through tuning of the structural and electronic properties at an atomic level of the ETL for thin film perovskite devices with a total thickness of about $600 \mathrm{~nm}$. However, it is a huge challenge to integrate thin ETL films with other materials into one single electronic device, within a large area and with a sufficient yield. Additionally, the stability issues of PSCs might not be caused by the charge transporting layers.

\subsection{Transient decay technology}

Transient photo-voltage/photo-current decay (TPV/TPC) measurements can provide useful information on charge transfer properties of the dye-sensitized solar cells, organic solar cells and PSCs. ${ }^{76,77}$ For the PSCs, there might be different schematic diagrams of electron energy levels based on the device structures. For example, Green et al. suggested an energy-band diagram as shown in Fig. $8 \mathrm{a},{ }^{78}$ which was based on electrontransfer processes in perovskite nanoparticles and the HTL/ perovskite $/ \mathrm{TiO}_{2}$ device. ${ }^{79} \mathrm{Fig}$. $8 \mathrm{~b}$ shows a modified version of the band diagram for the commonly used structure of PSCs in which the electron affinities and band bending in the ITO and $\mathrm{SnO}_{2}$ ETL have been omitted.

Similar to EIS measurements, it is impossible for the transient measurements to provide information about the chemical properties of charge carrier species and interfacial recombination. However, the dominant electron transfer process in the device can be experimentally determined by tuning the charge collective layer. This is actually quite useful not only for the transient measurement but also for impedance characterization. For example, by tuning the charge collective layer in the PSCs, it is highly possible to distinguish the contribution from either the ETL or HTL. Fig. 9a and b illustrate the transient photovoltage decay and transient photocurrent decay measurement principles and the corresponding voltage decay signals for the PSCs. ${ }^{80}$

The idea of photo-transient measurement is to use a light transient of small amplitude to probe the device that is under equilibrium conditions, and then monitor the decay kinetics. In practice, this can be achieved by using a short-pulsed laser as

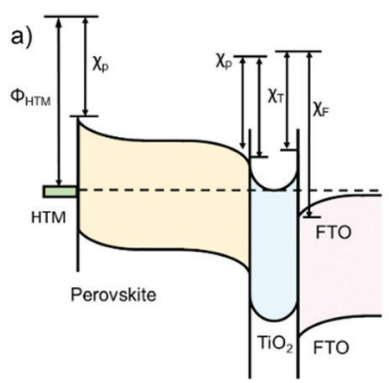

b)

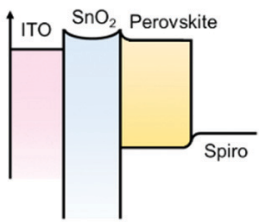

Fig. 8 (a) Energy-band diagram deduced from the vacuum energy levels $\chi_{\mathrm{P}}, \chi_{\mathrm{T}}$ and $\chi_{\mathrm{F}}$ represent the electron affinities of the perovskite, $\mathrm{TiO}_{2}$ and FTO layers, respectively, and $\Phi_{\text {HTM }}$ represents the work function of the HTM layer. (Modified from ref. 78.) (b) The simplified energy-band diagram for the planar structured PSC. a)

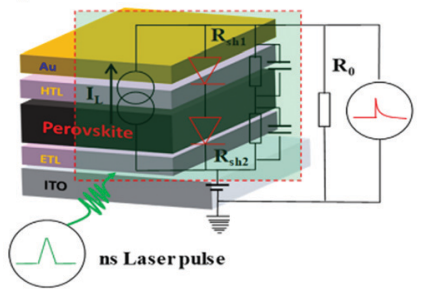

c)

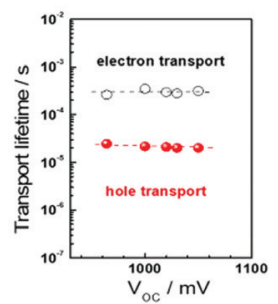

b)

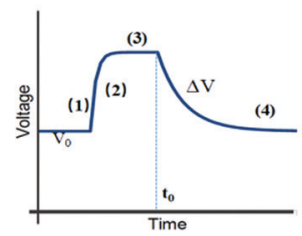

d)

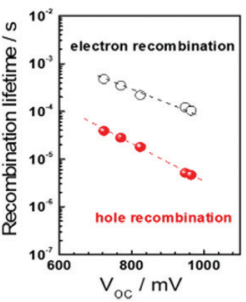

Fig. 9 ( $a$ and b) The working scheme of transient photovoltage/photocurrent decay measurement. (b) Shows transient photocurrent decay data for an example device. (c) Transport lifetime and (d) recombination lifetime results from using bi-exponential fitting of the transient photocurrent decay and transient photovoltage decay, respectively.

a probe to generate a voltage perturbation about the $V_{\text {OC }}$ of the cell under the white bias light produced by an array of white light emitting diodes and measuring the voltage decay process thereafter (under open circuit conditions). Specifically, a bias lamp is used for the PSC to maintain bias irradiation with a steady state voltage, $V_{0}$ (Fig. $9 \mathrm{~b}(1)$ ). Incident red light is superimposed with the previous white light under open-circuit conditions, which boosts the voltage, $V=V_{0}+\Delta V$, (Fig. $9 \mathrm{~b}(2)$ and (3)). The red light is turned off at $t=t_{0}$. Then the photovoltage begins to decay, and returns to the steady state (Fig. 9b(4)). In this case, all the charge carriers generated by the light pulse are assumed to be recombined. When it is excited with a short pulse of light (as short as nano-seconds for the measurement of the PSCs), the photo-generated charges are extracted on the electrodes, thus resulting in a current which can be detected using an oscilloscope in the form of voltage across a resistor (under short circuit conditions). In this case, all the charge carriers generated by the light pulse are assumed to be collected. Transient photocurrent decay measurements are generally conducted under short circuit conditions and provide information about extractable charges, charge recombination and density of states.

In order to precisely obtain the carrier transport parameters based on the photocurrent/photovoltage decay measurements, it is critical to consider the energy band bending effect at the junctions in the PSC devices, i.e., in short circuit or open circuit situations. Fig. 10 shows the electron energy levels of the conduction band and valence band during the transient decay measurements, in which the Fermi levels for an electron in the ETL (and for hole in the HTL) and in the perovskite layer are equal under open circuit conditions for photovoltage decay measurements. However, there is a band-bending for the electron energy levels in the ETL, HTL and perovskite layers in the case of short circuit conditions. The Fermi levels for 
Open Circuit

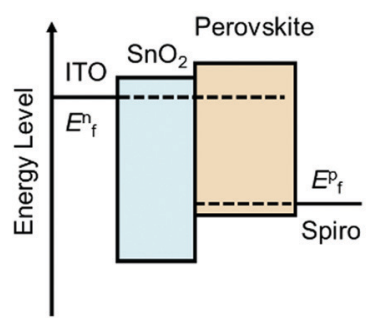

Short Circuit

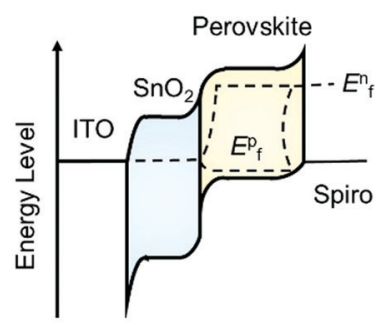

Fig. 10 Electron energy levels at open circuit (left) for the transient photovoltage decay measurement and at short circuit (right) for the transient photocurrent decay measurement on a PSC using $\mathrm{SnO}_{2}$ and spiro-OMeTAD as ETL and HTL. The dotted lines indicate the Fermi level of electrons and holes in the perovskite layer.

electrons in the ETL and holes in the HTL for the case under open circuit conditions are different from those in the short circuit case. Therefore, either a bias of voltage or current is needed to apply onto the device to offset the photovoltage/ photocurrent produced by steady-state illumination during the transient photovoltage/photocurrent decay measurements. When using this transient decay technique to characterize the PSCs, the bi-exponential decay trends of transient photovoltage and transient photocurrent can often be observed for the signals of electron/hole transport or recombination lifetimes (Fig. 9c and d).

Combining the energy-band diagram shown in Fig. 10 and the ambipolar transport properties of perovskite, we suggested electron/hole transport or recombination lifetimes are the key characteristics of electrons and holes at different time scales. ${ }^{81}$ For example, the electron and hole diffusion lengths were reported to be $177 \mathrm{~nm}$ and $813 \mathrm{~nm}$ for the $\mathrm{FAPbI}_{3}$ $\left(\mathrm{FA}=\mathrm{HC}\left(\mathrm{NH}_{2}\right)_{2}\right)$, whereas a mixed-ion perovskite $\left(\mathrm{MAPbI}_{3-x} \mathrm{Cl}_{x}\right)$ has shown an opposite situation and they were $1069 \mathrm{~nm}$ and $1213 \mathrm{~nm}$ for electrons and holes, respectively. ${ }^{82}$ This indicates that electrons and holes are transported at different rates in this ambipolar material and they have different surface recombination velocities. This is easily understood because the ambipolar material (e.g., perovskite), electron transporting layer $\left(\mathrm{SnO}_{2}\right.$ or $\left.\mathrm{SnS}_{2}\right)$ and hole transporting layer (spiroOMeTAD) all possess different electron affinities as discussed above. This can result in different charge transport properties and thus they have different charge transfer lifetimes and recombination lifetimes. ${ }^{83}$ Therefore, by performing transient photovoltage/photocurrent decay characterization in subnanosecond scales at an open-circuit/short circuit, the charge carrier recombination lifetime $\left(\tau_{\mathrm{n}}\right)$ and transport lifetime $\left(\tau_{\mathrm{tr}}\right)$ at the ETL and HTL for electrons and holes can be empirically evaluated by fitting the obtained transient signals. ${ }^{84,85}$

The mobilities of electrons and holes for the ETL and HTL are different due to the difference in their surface states, which is dependent on the materials used in the system. For instance, for the PSCs with the ETL using $\mathrm{SnO}_{2}$ or $\mathrm{TiO}_{2}$ and HTL using spiro-OMeTAD doped with Li salt (lithium bis(trifluoromethanesulfonyl)imide) and TBP (4-tert-butylpyridine), the hole mobility was found to be slightly higher than that of electrons. ${ }^{86}$
The transient photocurrent decay signals can be fitted bi-exponentially, and a shorter transport lifetime corresponding to the holes and a longer transport lifetime corresponding to the electrons could be easily obtained. The recombination lifetime could be also caused by the difference in the numbers of carriers during their recombination. We reported that the short lifetime was related to the charge carrier recombination at the perovskite/spiro-OMeTAD interface, whereas the long lifetime to the charge confined at the perovskite/ETL interface. ${ }^{87}$ The charge carrier diffusion coefficient can be calculated according to eqn (3):

$$
D_{\mathrm{n}}=\frac{d^{2}}{C_{1} \times \tau_{\mathrm{tr}}}
$$

where $d$ is film thickness, $D_{\mathrm{n}}$ is carrier diffusion coefficient, $\tau_{\mathrm{tr}}$ is transport time, and $c$ is a constant of 2.35 , correlated with the charge conduction mechanism. A hopping mechanism, rather than a band conduction mechanism, would usually take place in the nanocrystalline semiconducting films if the perovskite materials are considered as having defect intolerant properties. The $\tau_{\text {tr }}$ values of both the electrons and holes can be obtained from the transient photocurrent decay measurement, separately. The diffusion lengths of electrons and holes can then be evaluated according to eqn (4):

$$
L_{\mathrm{n}}=\sqrt{D_{\mathrm{n}} \times \tau_{\mathrm{n}}}
$$

where $L_{\mathrm{n}}$ is carrier diffusion length.

\section{Advanced technologies in ETLs for PSCs}

\subsection{Design rules for ETLs}

The specific design rules of ETLs for improving photovoltaic parameters are discussed in this section. Firstly, the $J-V$ curve (in light and dark conditions) of heterojunction PSCs can be described using: ${ }^{88}$

$$
\ln \left(J_{\mathrm{SC}}-J-\frac{V}{R_{\mathrm{Sh}}}\right)=\frac{q}{\left(m_{1}+m_{2}\right) K T}\left(V+J R_{\mathrm{s}}\right)+\ln J_{0}
$$

where $R_{\mathrm{s}}$ and $R_{\mathrm{sh}}$ are the series resistance and shunt resistance, respectively. $m_{1}$ and $m_{2}$ are the diode ideality factors of the ETL/perovskite and perovskite/HTL p-n junctions, respectively. $R_{\mathrm{s}}, R_{\mathrm{sh}}, J_{0}$ and $m$ can be numerically obtained by simulating the $J-V$ curves (both in light and dark) of the PSCs. We have considered a typical PSC using an active layer with a bandgap of $\sim 1.6 \mathrm{eV}$. The theoretical maximum $V_{\mathrm{OC}}$ and $J_{\mathrm{SC}}$ of such solar cells, calculated using the detailed balance model ( $\mathrm{S}-\mathrm{Q}$ limit), are $1.32 \mathrm{~V}$ and $25 \mathrm{~mA} \mathrm{~cm}{ }^{-2}$, respectively. ${ }^{147}$ The working devices generally achieve a $V_{\mathrm{OC}}$ value of $1.1 \mathrm{~V}$, and a $J_{\mathrm{SC}}$ value of $22 \mathrm{~mA} \mathrm{~cm}{ }^{-2}$ according to most reports. In addition, the series resistance under illumination conditions could be estimated: $R_{\mathrm{s}, \text { light }}=\left(V_{\mathrm{OC}, \text { theory }}-V_{\mathrm{OC}}\right) / J_{\mathrm{SC}}=(1.32-1.1 \mathrm{~V}) / 22 \mathrm{~mA} \mathrm{~cm}{ }^{-2}=$ $10 \Omega \mathrm{cm}^{2}$. In most the cases, the dark series resistance $R_{\mathrm{S}}$ accounts for half of the light series resistance $R_{\mathrm{s}, \text { light }}$, and thus the $R_{\mathrm{S}}$ value is estimated to be $\sim 5 \Omega \mathrm{cm}^{2}{ }^{149} \mathrm{~A}$ smaller $R_{\mathrm{S}}$ value 
below $5 \Omega \mathrm{cm}^{2}$ is beneficial for obtaining higher values of $V_{\mathrm{OC}}$ and fill factor (FF), which is in accordance with the report in the literature. ${ }^{150}$ The ionic-covalent nature of hybrid halide lead perovskites leads to interactions with the ETL with functional groups. For example, doping or chemically modifying the interface between the perovskite crystal and ETL could have contributed to efficient electron extraction and electron transport, and thus to an enhanced $J_{\mathrm{SC}}$.

Secondly, the $V_{\mathrm{OC}}$ values of PSCs depend on exciton dissociation efficiency, charge transport and the effective density of states according to eqn (6): ${ }^{89}$

$$
V_{\mathrm{OC}} \propto \frac{E_{\mathrm{gap}}}{q}-\frac{A k T}{q} \ln \left(\frac{(1-P) \gamma N_{\mathrm{c}}^{2}}{P G}\right)
$$

where $\gamma$ is the Langevin recombination constant, $P$ the dissociation probability of a bound electron-hole pair into free charge carriers, $G$ the generation rate of bound electron-hole pairs, $N_{\mathrm{c}}$ the effective density of states, and $A$ the exponential factor. Therefore, for the ETL, it is necessary to reduce the interfacial charge accumulation by enabling a long electron diffusion length and a balanced charge transport. As a result, this allows for less non-radiative recombination opportunities at the ETL/perovskite interface and could contribute to the augmented $V_{\text {OC: }}$

$$
V_{\mathrm{OC}}=\frac{E_{\mathrm{g}}}{e}-\frac{K T}{e} \ln \frac{N_{\mathrm{c}} N_{\mathrm{v}} \beta}{G}
$$

where $G$ equals the non-radiative recombination rate. ${ }^{90}$

Thirdly, the FF of the PSCs is mainly affected by the series resistance $R_{\mathrm{S}}$ of the device and thus a low value of $R_{\mathrm{S}}$ is necessary to eliminate the potential charge loss at high bias voltages and achieve a high FF. ${ }^{91,92}$ As for the ETL, it is critical to form a smooth surface with less defects, in order to reduce the charge losses and maintain $R_{\mathrm{s}}$ smaller than $5 \Omega \mathrm{cm}^{2}$. Consequently, a high value of FF could be obtained along with a high PCE $>20 \%$. Theoretical analysis based on the ShockleyQueisser efficiency limit showed that the FF for an efficient single junction device can reach as high as $\sim 90 \%$ at room temperature. $^{93}$

\subsection{Electron transport materials in PSCs}

Recently, great effort has been made to obtain a high PCE by modifying the ETLs or designing new electron transport materials. In 2015, Liu et al. introduced a self-assembled silane monolayer between the $\mathrm{TiO}_{2}$ and $\mathrm{CH}_{3} \mathrm{NH}_{3} \mathrm{PbI}_{3}$, achieving optimized interface band alignments and enhanced charge lifetime. The silane monolayer helps tune the interfacial electronic structures and passivate the recombination process, and thus a high efficiency of $12.7 \%$ was obtained for a $\mathrm{TiO}_{2} /$ $\mathrm{SAM} / \mathrm{CH}_{3} \mathrm{NH}_{3} \mathrm{PbI}_{3}$-based mesoscopic solar cell prepared using a sequential deposition method. ${ }^{94}$ In 2016, Zuo et al. reported that deposition of various self-assembled monolayers with different functional groups onto the $\mathrm{SnO}_{2}$ surface resulted in chemical interactions within the perovskite layer. The surface trap states were suppressed and the charge transfer at the perovskite/ $\mathrm{SnO}_{2}$ interface was enhanced due to the decreased work function and improved interfaces between perovskite and $\mathrm{SnO}_{2}$. The device's efficiency was increased up to $18.8 \%$, showing a $10 \%$ improvement compared to that without using the self-assembled monolayer. ${ }^{95}$ The above work highlights the importance of surface modification and chemical interactions at the perovskite/ETL interfaces, which paves the way for further optimizing ETL development for the PSCs.

$\mathrm{TiO}_{2}$ has been used as an ETL due to its suitable energy levels of conduction band minimum (CBM $\sim-3.9 \mathrm{eV})$ and valence band maximum $(\mathrm{VBM} \sim-7.2 \mathrm{eV}) .{ }^{75}$ One of the major disadvantages for the $\mathrm{TiO}_{2}$ ETL is its high processing temperature, which is associated with high costs and unsuitability for flexible PSCs. The electron recombination in the $\mathrm{TiO}_{2}$ ETL is also quite significant due to its inferior electron mobility compared to that of the perovskite, thus resulting in unbalanced charge transfer and consequently poor performance and low efficiency. Moreover, it also suffers from low conductivity and carrier accumulation owing to its numerous trap states. ${ }^{96,97}$

$\mathrm{SnO}_{2}$ has been regarded as a good ETL material with its advantages including low chemical reactivity and photocatalytic activity, ${ }^{98}$ a wider band gap of $\sim 3.8 \mathrm{eV},{ }^{99}$ a higher mobility ${ }^{100,101}$ and a deeper conduction band $(-4.3 \mathrm{eV})^{102}$ than those of $\mathrm{TiO}_{2}$, which in principle should facilitate more efficient electron transfer from the perovskite. However, most of the devices based on low-temperature solution-processed $\mathrm{SnO}_{2}$ were reported to suffer from serious photocurrent hysteresis which makes it difficult to determine their real PCEs. ${ }^{103,104}$

The optoelectronic properties of n-type semiconducting $\mathrm{ZnO}$, such as a suitable conduction band energy level ( $\sim-4.17 \mathrm{eV}$ for the CBM), large optical bandgap of $3.3 \mathrm{eV}$, high electronic mobility and high transmittance, are promising for applications in PSCs. ${ }^{105-107}$ In addition, the low-temperature processed $\mathrm{ZnO}$ nanocrystalline thin films are favourable for flexible devices. ${ }^{64}$ Nevertheless, $\mathrm{ZnO}$ could cause decomposition of organometal halide perovskites, which may impede its largescale application in PSCs. ${ }^{63}$

$\mathrm{Nb}_{2} \mathrm{O}_{5}$ is a wide bandgap n-type semiconductor with a good chemical stability, an excellent optical transmittance and a higher CBM $(-4.33 \mathrm{eV})^{108}$ than that of $\mathrm{TiO}_{2}$, which has been applied as an ETL of the PSCs. ${ }^{109,110}$ For example, Feng et al. deposited amorphous $\mathrm{Nb}_{2} \mathrm{O}_{5}$ ETL for large-area rigid PSCs and flexible planar PSCs using an e-beam evaporation method, obtaining PCEs of $18.59 \%$ and $15.56 \%$, respectively. ${ }^{111}$ Moreover, other binary metal oxides, such as WOx ${ }^{112,113}$ and $\mathrm{In}_{2} \mathrm{O}_{3},{ }^{114,115}$ and ternary metal oxides, such as $\mathrm{Zn}_{2} \mathrm{SnO}_{4},{ }^{116,117}$ $\mathrm{BaSnO}_{3}{ }^{118,119}$ and $\mathrm{SrTiO}_{3},{ }^{120,121}$ have been proposed for highefficiency PSCs as listed in Table 1.

Based on eqn (2), the free electron concentration is dependent on the charge trapping-de-trapping process via defects in the metal oxide nanocrystals. ${ }^{122}$ Therefore, an attenuated electron conductivity for most of the conductive metal oxide nanocrystals is usually observed due to the existence of defects inside. Consequently, several strategies have been proposed to increase the conductivity by increasing the number of free electrons and/or diffusion coefficients by doping and alloying engineering. ${ }^{123}$ For example, doping $\mathrm{SnO}_{2}$ and $\mathrm{TiO}_{2}$ ETLs with 
Table 1 Electronic properties of ETLs for PSCs

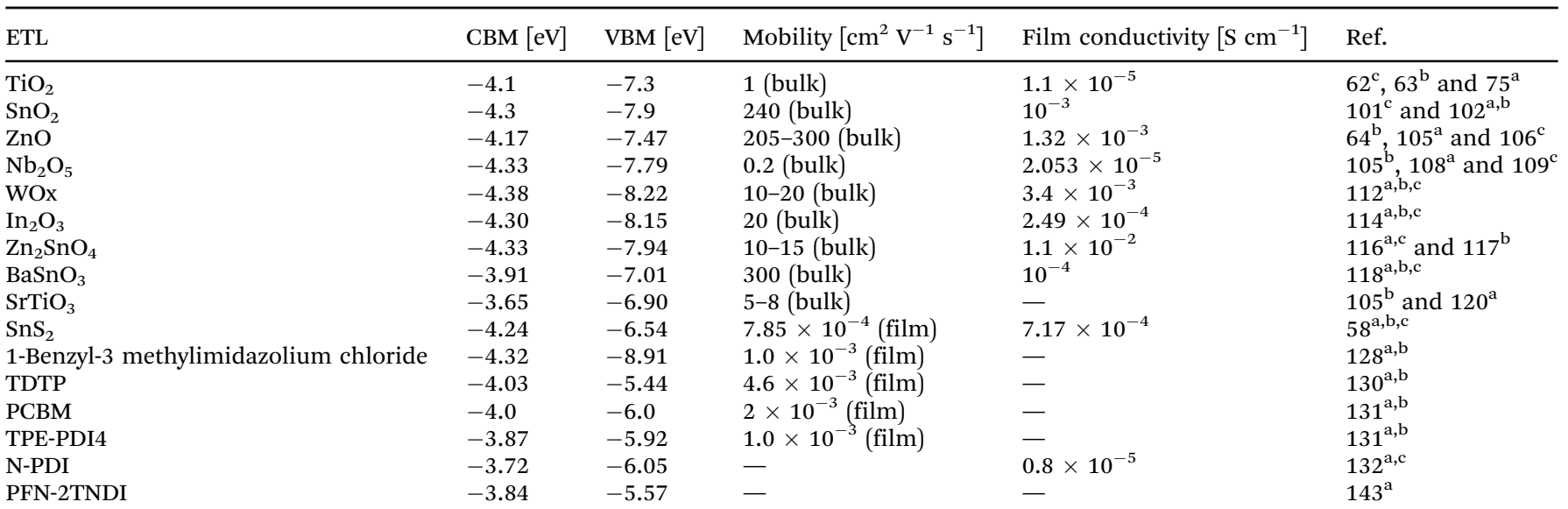

The labels a, b and c correspond to the references of energy levels (CBM and VBM), mobility and film conductivity, respectively.

metal cations $\left(\mathrm{Y}^{3+}, \mathrm{Al}^{3+}, \mathrm{Mg}^{2+}, \mathrm{In}^{+}\right)$has significantly increased the diffusion coefficient to the range of $10^{-5}-10^{-4} \mathrm{~cm}^{2} \mathrm{~s}^{-1}$ and thus has enhanced the electrical conductivity of the ETLs to $\sim 10^{-4} \mathrm{~S} \mathrm{~m}^{-1}$. $^{124-127}$

Ionic liquids (IL) have also been introduced into PSCs as ETLs or interface modifiers due to their good thermal and electrochemical stability, high electrical conductivity and carrier mobility. For example, Yang et al. used solid state ionic-liquid (ss-IL), (1-benzyl-3 methylimidazolium chloride) as the ETL, prepared using a low-temperature solution process. ${ }^{128}$ The hydrophilic nature of ss-IL facilitates the formation of highquality perovskite films with reduced electron trap-state densities. The flexible PSC has achieved a record PCE of $16.09 \%$. Meanwhile, they developed 1-butyl-3-methylimidazolium tetrafluoroborate IL to tailor the surface properties of $\mathrm{TiO}_{2}$, including smoothening the $\mathrm{TiO}_{2}$ surfaces, reducing the work function of $\mathrm{TiO}_{2}$, and improving 0electron mobility. ${ }^{129}$ After modification by the IL, the device PCE was increased to $19.62 \%$, and the $J-V$ hysteresis was completely eliminated due to significantly reduced traps and balanced charges.

Recently, many organic small molecules have been developed as the ETLs for highly efficient PSC devices. Compared with their inorganic counterparts, the organic ETLs offer fascinating flexibility of chemistry to react with perovskite compounds and low processing temperature for device fabrication. For example, $\mathrm{Gu}$ et al. proposed an n-type sulfur-containing azaacene (TDTP) as the ETL to replace the PCBM in an inverted PSC. ${ }^{130}$ The sulphur species inside the molecular structures was identified to dramatically improve the interfacial interactions between the ETL molecules and perovskite layer through formation of $\mathrm{S}-\mathrm{I}$ or $\mathrm{S}-\mathrm{Pb}$ chemical bonds, thus displaying a high electron mobility of $4.6 \times 10^{-3} \mathrm{~cm}^{2} \mathrm{~V}^{-1} \mathrm{~s}^{-1}$ and passivating the perovskite surface, which enhances the PCE up to $18.2 \%$ (Fig. 11a). Jiang et al. synthesized three-dimensional perylenediimide (PDI)-based molecules (TPE-PDI4) with a high electron mobility of $1.0 \times 10^{-3} \mathrm{~cm}^{2} \mathrm{~V}^{-1} \mathrm{~s}^{-1}$. When the TPEPDI4 was used as the ETL in their study, the obtained PSCs achieved a high PCE of $16.29 \%$ (Fig. 11b). Moreover, when used as a thin interfacial layer, the PSCs based on TPE-PDI4 could achieve an efficiency of $18.78 \% .^{131}$

Besides, a few studies have fully demonstrated the overall advantages of using these organic ETLs, including adjustability of optoelectronic properties by structural changes, availability of multi-defined structures, and easy purification and synthesis in material processing, as well as batch-to-batch reproducibility and flexibility. For example, Zhang et al. introduced an aminosubstituted perylene diimide derivative (N-PDI) ETL into the PSCs. ${ }^{132}$ The interactions between the amino groups of N-PDI and Sn(Iv) of the FTO substrate have been found to accelerate the surface electron extraction and passivate the surface trap states of the $\mathrm{MAPbI}_{3-x} \mathrm{Cl}_{x}$ film by using the N-PDI (Fig. 11c). Owing to the significant reduction of electron trap density by passivation, a smaller capacitance was obtained from the response in the intermediate frequency region in the EIS results for the N-PDI based device. This decrease in capacitance results

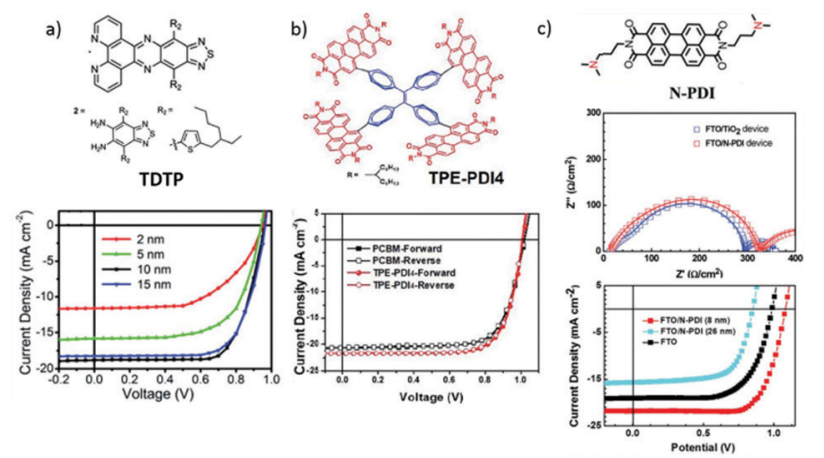

Fig. 11 (a) Molecular structure of TDTP and J-V curves of PSCs with different TDTP thicknesses. ${ }^{130}$ (b) Chemical structure of TPE-PDI4 and forward/reverse scans of PSCs using TPE-PDI4 and PCBM as ETLs, respectively. ${ }^{131}$ (c) Molecular structure of N-PDI, EIS curve of PSCs with different ETLs obtained at around $V_{O C}$ under illumination with an LED array emitting white light, the applied voltage of $0.9 \mathrm{~V}$ is given, and $J-V$ curves of the perovskite solar cells based on FTO/N-PDI with different thicknesses and the control device based on the FTO substrate. ${ }^{132}$ Reproduced from ref. 130-132 with permission from the Royal Society of Chemistry. 


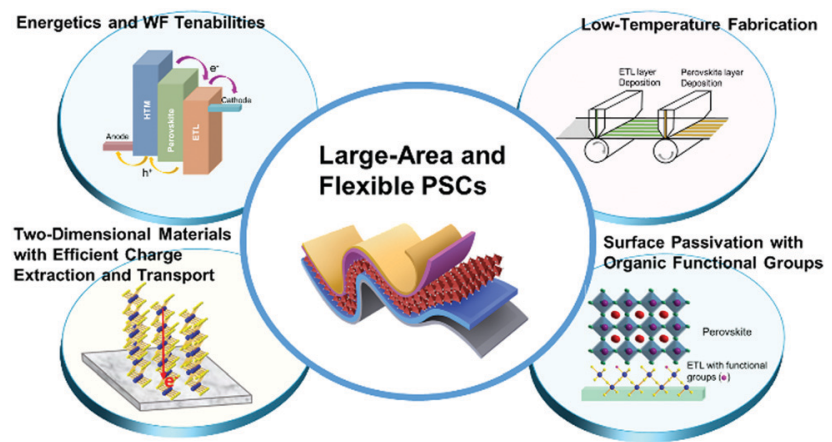

Fig. 12 Outlines for the design features and development trend of ETLS for the large area and flexible PSCs, including the energetics and work function tenability between the ETL and perovskite layer, the methods for low-temperature fabrication of various functional layers, and the strategies for efficient charge extraction and transport via two-dimensional materials or surface passivation with organic functional groups for instance.

in a higher $V_{\mathrm{OC}}$ value and boosts the $J_{\mathrm{SC}}$ and $\mathrm{FF}$ for N-PDI-based devices. As a result, an excellent PCE of $17.66 \%$ on the FTO substrate was achieved. ${ }^{132}$

In addition, double-layer ETLs are also used to further improve the charge extraction and passivate the surface defects of the perovskite. For example, $\mathrm{TiO}_{2} / \mathrm{SnO}_{2}$ bilayer ETLs can reduce the leakage current and the charge recombination compared with a single ETL of either $\mathrm{TiO}_{2}$ or $\mathrm{SnO}_{2}{ }^{133}$ Wang et al. found that [6,6]-phenyl-C61-butyric acid (PCBA) could partially fill the surface trap states of $\mathrm{SnO}_{2}$ and passivate the interfaces between $\mathrm{SnO}_{2}$ and perovskite in the $\mathrm{SnO}_{2} / \mathrm{PCBA}$ based bilayer PSCs. ${ }^{134}$ Moreover, a $\mathrm{ZnO/fullerenes} \mathrm{bilayer}$ ETL could efficiently hinder the reactions between $\mathrm{ZnO}$ and the perovskite during annealing $\left(>100{ }^{\circ} \mathrm{C}\right)$, thus improving the device's stability. ${ }^{135}$

The electron selective layers of perovskite solar cells are also critical for achieving good photovoltaic properties. ${ }^{136}$ Despite exhibiting a low electron mobility for the a-SnO $\mathrm{S}_{2}$ (generally prepared using a sol-gel method), its suitable electronic energies combined with high transparency $\left(E_{\text {gap }}>4 \mathrm{eV}\right)$ and uniform substrate coverage make it an excellent ETL candidate for the low-cost and large-scale fabrication of organo-halide lead PSCs and other organic photovoltaics. Fig. 12 outlines the design features and development trends of ETLs for large area and flexible PSCs, including the energetics and work function tenability between the ETL and perovskite layer, the method for low-temperature fabrication of various functional layers, and the strategies for efficient charge extraction and transport via two-dimensional materials or surface passivation with organic functional groups for instance.

For example, Shin et al. reported a low-temperature $\left(<180{ }^{\circ} \mathrm{C}\right)$ solution-processed $\mathrm{SnO}_{2}$ ETL prepared using a spin-coating method, which showed good antireflection, suitable band edge positions, and high electron mobility. ${ }^{137}$ As a result, the planar PSC made using this layer has achieved a PCE of $17.21 \%$ with a high $V_{\mathrm{OC}}$ value of $1.11 \mathrm{~V}$ and a high $J_{\mathrm{SC}}$ value of $23.27 \mathrm{~mA} \mathrm{~cm} \mathrm{~cm}^{-2}$ under the reverse voltage scan. Yang et al. applied amorphous $\mathrm{TiO}_{2}\left(\mathrm{am}-\mathrm{TiO}_{2}\right)$ as the ETL, which can be
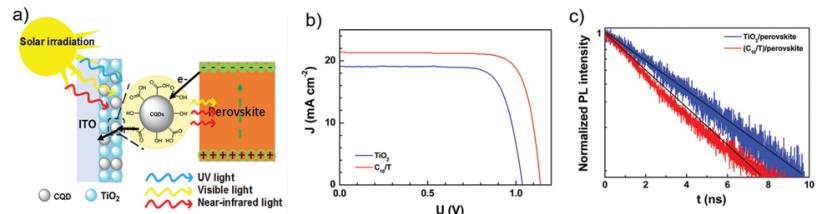

d)
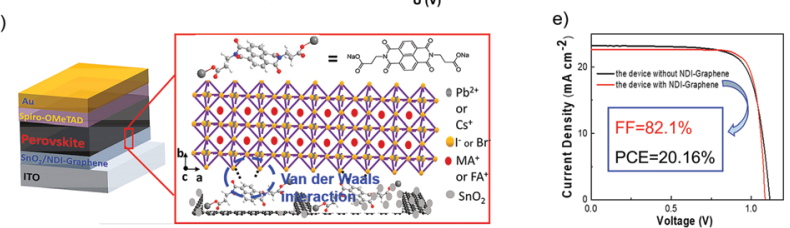

Fig. 13 (a) Schematic illustration of the working mechanism for the perovskite with CQD-doped $\mathrm{TiO}_{2}$ as the ETL. (b) $J-V$ curves of PSCs based on ITO/C1O/T and the control device based on the ITO/ $\mathrm{TiO}_{2}$ substrate. (c) Time-resolved $\mathrm{PL}$ decay transients for $\mathrm{TiO}_{2} /$ perovskite (black), and CQD-doped $\mathrm{TiO}_{2}$ /perovskite (red) films. (d) Schematic architecture of planar perovskite solar cells. ${ }^{66}$ The expanded scheme shows the chemical structure of NDI and the bonding conditions of NDI-graphene with perovskite films. (e) $J-V$ curve (reverse scan) of device based on $\mathrm{SnO}_{2}-5 \%$ G ETL. ${ }^{141}(\mathrm{a}-\mathrm{c})$ Reproduced with permission from ref. 66. Copyright 2017 American Chemical Society. (d and e) Reproduced from ref. 141. Copyright 2018 American Chemical Society.

prepared at room temperature using a magnetron sputtering method. ${ }^{138}$ The film exhibited favourable transmittance and fast electron extraction and transport. The large-area $\left(>10 \mathrm{~mm}^{2}\right)$ and flexible PSCs based on $\mathrm{am}_{-\mathrm{TiO}}$ achieved a PCE of $15.07 \%$. Li et al. recently introduced homogeneous carbon quantum dots (CQDs) into the nanocrystal $\mathrm{TiO}_{2}$ solution to form a high quality ETL for efficient PSCs (Fig. 13a). ${ }^{66}$ Notably, these CQDs exhibited excellent light harvesting ability and acted as an electron reservoir, thus leading to a significant improvement of efficient charge carrier extraction and injection between the $\mathrm{TiO}_{2}$ and perovskite layer in PSCs. When the amount of CQDs added was $10 \mathrm{wt} \%$, the planar heterojunction PSC achieved a PCE as high as 19\% (Fig. 13b). This is mainly attributed to the improvement of electron mobility and electron extraction ability (Fig. 13c), and the good matching of the energy levels between the ETL and perovskite active layer by the adding these CQDs. All of these have led to remarkable increases in both the values of $J_{\mathrm{SC}}$ and $V_{\mathrm{OC}}$.

The electronic conductivity of $\mathrm{SnO}_{2}$ can also be increased via doping a moderate level of graphene quantum dots (GQDs). ${ }^{139}$ Therefore, photo-generated electrons transferred from the GQDs to $\mathrm{SnO}_{2}$ could effectively fill electronic traps and thus improve the device's PCE from $17.91 \%$ to $20.31 \%$ with significantly reduced hysteresis. A further thermal treatment of dispersions of GQDs and $\mathrm{SnO}_{2}$ has been found to improve the film coverage and reproducibility. ${ }^{140}$

Zhao et al. reported the usage of electronic conducting graphene to form graphene/ $\mathrm{SnO}_{2}$ composites, which were then used as an ETL in the PSC (Fig. 13d). ${ }^{141}$ Van der Waals interactions were generated between the perovskite material and graphene, which were proved to be able to fix the octahedral $\left[\mathrm{PbI}_{6}\right]^{4-}$ at the perovskite/ETL interface. After optimization, a high PCE of $20.2 \%$ and a high FF of $82 \%$ have been achieved by using $\mathrm{FA}_{0.75} \mathrm{MA}_{0.15} \mathrm{Cs}_{0.1} \mathrm{PbI}_{2.65} \mathrm{Br}_{0.35}$ perovskite and the optimized amount of graphene of $5 \mathrm{v} / \mathrm{v} \%$. This good performance 
has been attributed to the enhancement of electron mobility and extraction ability, and the reduced carrier recombination (Fig. 13e).

Yang et al. mixed ethylene diamine tetra-acetic acid (EDTA) into a $\mathrm{SnO}_{2}$ aqueous colloidal dispersion to prepare an EDTAcomplexed $\mathrm{SnO}_{2}\left(\mathrm{E}-\mathrm{SnO}_{2}\right)$ ETL. ${ }^{100}$ Compared to that of $\mathrm{SnO}_{2}$, this $\mathrm{E}-\mathrm{SnO}_{2}$ showed a smaller contact angle with water, thus resulting in the reduced Gibbs free energy for the nucleation of perovskites and lowered surface energy for its growth. All these resulted in the production of high-quality perovskite films. Moreover, the EDTA modification increased the electron mobility by three times, suppressed hysteresis, and improved the $V_{\text {OC }}$ value due to an improved energy level alignment against perovskite. A rigid PSC and a flexible PSC with the $\mathrm{E}-\mathrm{SnO}_{2}$ as the ETL achieved PCEs of $21.60 \%$ and $18.28 \%$, respectively.

Gong et al. reported the use of F-doped $\mathrm{SnO}_{2}\left(\mathrm{~F}: \mathrm{SnO}_{2}\right)$ nanocrystals as the ETL in an n-i-p planar PSC (Fig. 14a), and the device's $V_{\mathrm{OC}}$ values could be tailored by gradually changing the band offset at the interface of the perovskite active layer and ETL. The up-shifts of the Fermi level of the new ETL by doping of $\mathrm{F}^{-}$were reported to be around 68 and $95 \mathrm{meV}$, which are beneficial for free electron extraction and transport. The built-in potential at the ETL/perovskite heterojunction interface was estimated to be $1.06 \mathrm{~V}$, which can effectively decrease the carrier recombination and increase the $V_{\mathrm{OC}}$. Consequently, the $\mathrm{n}-\mathrm{i}-\mathrm{p}$ planar PSC using this bilayer ETL has achieved a PCE of $20.2 \%$ with a high $V_{\text {OC }}$ of $1.13 \mathrm{~V}$ (Fig. 14b). ${ }^{142}$ Zhao et al. recently demonstrated a new strategy of utilizing a two-dimensional $\mathrm{SnS}_{2}$ as the ETL material for high-efficiency PSCs via a self-assembly stacking deposition method (Fig. 14c). The large-scale two-dimensional multilayer $\mathrm{SnS}_{2}$ sheet structure promoted heterogeneous nucleation over the perovskite precursor film. The intermolecular $\mathrm{Pb}-\mathrm{S}$ interactions between perovskite and $\mathrm{SnS}_{2}$ could passivate the a)

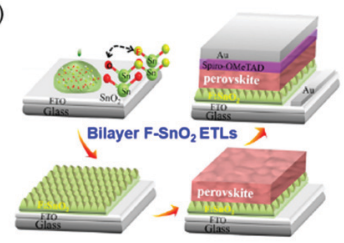

c)

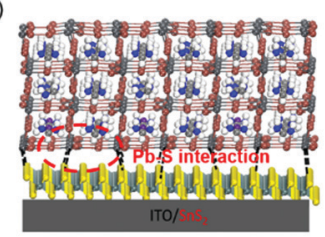

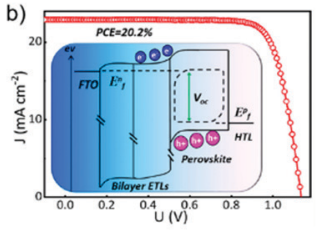

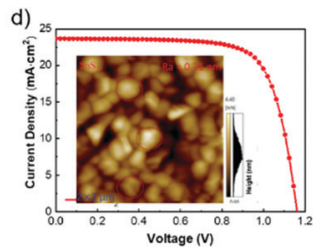

Fig. 14 (a) Flowchart of the fabrication process. (b) $J-V$ curves of PSCS based on bilayer ETL; the inset is the Quasi-Fermi level splitting of the bilayer ETL based devices at open circuit under illumination. ${ }^{142}$ (c) Illustration of the perovskite films deposited on the $\mathrm{SnS}_{2}$ substrate. (d) J-V curves (reverse scan) of devices $\mathrm{A}\left(\mathrm{SnO}_{2}\right.$ as $\left.\mathrm{ETL}\right)$ and $\mathrm{B}\left(\mathrm{SnS}_{2}\right.$ as $\left.\mathrm{ETL}\right)$, respectively; the inset shows AFM images of a $2 \mathrm{D}$ multilayer $\mathrm{SnS}_{2}$ film. ${ }^{58}$ (a and $\mathrm{b}$ ) Reproduced with permission from ref. 142. Copyright 2018 American Chemical Society. (c and d) Reproduced with permission from ref. 58 . Copyright 2018 Wiley.
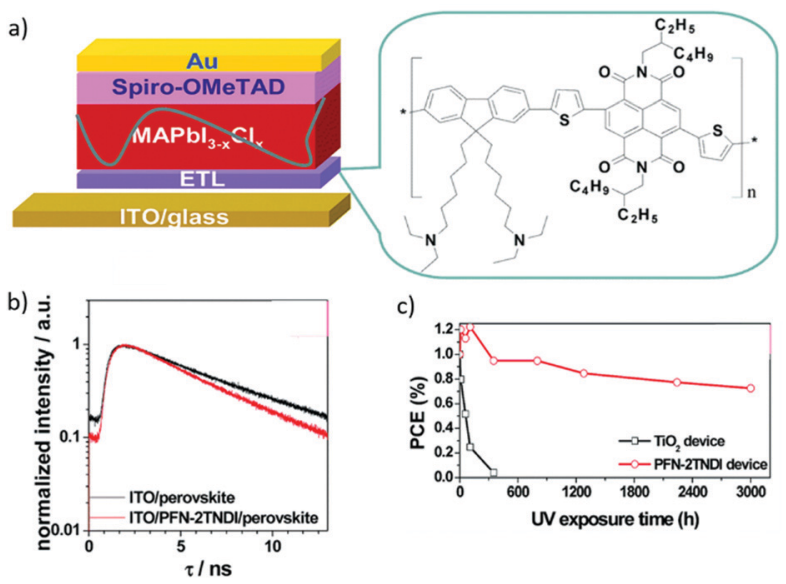

Fig. 15 (a) The $n-i-p$ structured planar heterojunction PSCs using the PFN-2TNDI electron transport layer. (b) Time-resolved PL decay of MAPbl $_{3-x} \mathrm{Cl}_{x}$ films on the ITO and ITO/PFN-2TNDI. (c) The evolution of PCE as a function of testing time under UV illumination for the PFN2TNDI- and $\mathrm{TiO}_{2}$-based PSCs. The devices were stored under exposure to UV in a $\mathrm{N}_{2}$-filled glove-box during testing. ${ }^{143}$ Reproduced from ref. 143 with permission from the Royal Society of Chemistry.

interfacial trap states, which suppressed charge carrier recombination and thus facilitated electron extraction for balanced charge transport at the interfaces between the electron transporting layer/perovskite and hole transporting layer/perovskite. Based on this design, a PCE value of $20.12 \%$ was realized for the 2D ETL based PSCs, along with a high $J_{\mathrm{SC}}$ of $23.55 \mathrm{~mA} \mathrm{~cm}^{-2}$ and an impressive $V_{\text {OC }}$ value of $1.161 \mathrm{~V}$ (Fig. 14d). ${ }^{58}$ The proposed $\mathrm{SnS}_{2}$ ETL has many advantages including the simple and convenient process, controllability, and enhanced contacts for charge transfer.

Polymer semiconductors possess many appealing features, some of which make them potentially good candidates for the ETL, such as simple process, adjustable energy levels and excellent optical and electrical properties. Generally, a semiconductor oxide can be conveniently dispersed in an organic phase to modify the surface area effect, volume effect and quantum size effect, and thus will lead to low carrier recombination rates. For example, Li et al. reported a copolymer PFN-2TNDI with a conjugated structure as the ETL in PSCs (Fig. 15a). ${ }^{143}$ The work function of the ITO was reduced to $3.81 \mathrm{eV}$ after the deposition of PFN-2TNDI $(5 \mathrm{~nm})$, thus facilitating formation of an ohmic contact and efficient charge transfer and extraction at the ETL/MAPbI ${ }_{3-x} \mathrm{Cl}_{x}$ interface (Fig. 15b). The PFN2TNDI device showed a good performance with a PCE value of $16 \%$. More importantly, in addition to the low temperature processability offered by the polymer ETL, the devices based on the polymer ETL showed significantly enhanced photostability against UV irradiation (Fig. 15c).

\section{Conclusion and outlook}

In this paper, we have comprehensively reviewed recent advances of ETLs based on organic and inorganic structures 
for highly efficient PSCs (PCE > 20\%). Unlike other thin film photovoltaics, production of perovskite solar cells in a large area becomes a significant issue for a successful commercialization, in which the methods of electron transport layers onto the transparent conductive oxide substrates becomes one of technique barriers. As discussed above, specific design methodologies of ETLs have been used to improve the photovoltaic parameters. Various properties (including electrochemical properties and surface morphology properties) of the ETLs have been exploited to enhance the performance of PSCs. An efficient ETL should possess high electron mobility, high electrical conductivity, uniform and pinhole-free film morphology and suitable LUMO energy levels, which should match with those of the perovskites. Besides, ultrafast spectroscopy characterization, electronic impedance spectroscopy characterization, and transient decay technology could provide critical information on carrier dynamics including charge extraction, diffusion and recombination related to the ETLs. With the help of these characterization techniques, significant enhancement of PCEs has been realized by designing new electron transport materials or modifying the ETLs. Metal oxides, for example $\mathrm{TiO}_{2}, \mathrm{SnO}_{2}$, and $\mathrm{ZnO}$, are the most widely used as ETLs. Further tailoring of their electrical properties can be realized by doping and alloying engineering or optimizing the surface of ETLs with lower defect density and forming a better contact with perovskites by introducing extra components. In addition, some organic molecules have been developed as electron transport materials.

Despite the rapid advances, further optimization of ETLs is critically desired in order to further improve the device efficiency and stability for successful commercialization. For the inorganic metal oxide-based ETLs, their components, synthesis or deposition methods, and post processes could be further optimized, for example, to lower the annealing temperature for crystallization or to optimise the morphology for the enhancement of electron transfer. For the organic ETLs, attention should be paid to the purity and stability under the heating treatment.

As the PSCs have already achieved excellent PCE values over $25 \%$, there should be further investigations for controlling factors and methods for the scaling up of large-area and flexible PSCs. First, it is well-known that the composition of the ETLs can largely impact the design of scalable deposition. ${ }^{29}$ Developing appropriate ETLs in order to meet the stringent requirements for large-area modules has become one of the crucial research topics. The ETLs should not only have high mobility, high conductivity, smoothness and compact coverage of film morphology and suitable energy level, but also be easily produced at a low-temperature $\left(<150{ }^{\circ} \mathrm{C}\right)$ in order to be compatible with the standard industrial processes as well. ${ }^{144}$

Secondly, a scalable deposition process should be capable of controlling nucleation and growth of perovskites, which requires advanced equipment and harsh production conditions. ${ }^{145}$ Strategies could be adopted to reduce fabrication technology requirements. For example, the surface energy and structure of the ETLs could be tailored by functionalizing them with surface-organic groups. Novel two-dimensional ETLs could also be designed in order to control the crystallization kinetics and film quality of perovskites.

Finally, PSCs fabricated using current scalable fabrication methods, including roll-to-roll production, doctor-blade coating and spray-coating, often show low PCEs and poor stability. To overcome such issues, hydrophobic groups such as fluorine and carbon nanotubes can be introduced into electron transport materials, which will not only improve the long-term stability of the PSCs against water, oxygen, light and heat, but also passivate surface defects of the ETLs to enhance the photovoltaic performance.

\section{Conflicts of interest}

There are no conflicts to declare.

\section{Acknowledgements}

The authors acknowledge financial support from the National Key Research and Development Program of China (2019YFE0101300), the Natural Science Foundation of China (NSFC No. 21673091, 51961165106, and 21975088), the NSFC Major International (Regional) Joint Research Project NSFCSNSF (No. 51661135023, and 51961165106), the Central Universities of Huazhong University of Science \& Technology (2018KFYXKJC034 and 2019kfyRCPY040), and the Double first-class research funding of China-EU Institute for Clean and Renewable Energy (3011187029), Newton Mobility (IE161019) through Royal Society and the NSFC. Reproduced from ref. 130132 and 143 with permission from the Royal Society of Chemistry. SA acknowledges funding from the European Union H2020 Programme under European Research council Consolidator grant [MOLEMAT, 726360].

\section{Notes and references}

1 P. Kung, M. Lin, P. Lin, J. Jhang, M. Pantaler, D. Lupascu, G. Grancini and P. Chen, Sol. RRL, 2020, 4, 1900306.

2 P. Li, X. Liu, Y. Zhang, C. Liang, G. Chen, F. Li, M. Su, G. Xing, X. Tao and Y. Song, Angew. Chem., Int. Ed., 2020, 59, 2-8.

3 L. Krückemeier, U. Rau, M. Stolterfoht and T. Kirchartz, Adv. Energy Mater., 2020, 10, 1902573.

4 H. Ren, S. Yu, L. Chao, Y. Xia, Y. Sun, S. Zuo, F. Li, T. Niu, Y. Yang, H. Ju, B. Li, H. Du, X. Gao, J. Zhang, J. Wang, L. Zhang, Y. Chen and W. Huang, Nat. Photonics, 2020, 14, 154-163.

5 J. Yoo, S. Wieghold, M. Sponseller, M. Chua, S. Bertram, N. Hartono, J. Tresback, E. Hansen, J. Correa-Baena, V. Bulović, T. Buonassisi, S. Shin and M. Bawendi, Energy Environ. Sci., 2019, 12, 2192-2199.

6 A. Solanki, P. Yadav, S. Turren-Cruz, S. Lim, M. Saliba and T. Sum, Nano Energy, 2019, 58, 604-611. 
7 D. Lin, T. Zhang, J. Wang, M. Long, F. Xie, J. Chen, B. Wu, T. Shi, K. Yan, W. Xie, P. Liu and J. Xu, Nano Energy, 2019, 59, 619-625.

8 C. Dong, X. Han, W. Li, Q. Qiu and J. Wang, Nano Energy, 2019, 59, 553-559.

9 Y. Liu, Q. Chen, H. Duan, H. Zhou, Y. Yang, H. Chen, S. Luo, T. Song, L. Dou, Z. Hong and Y. Yang, J. Mater. Chem. A, 2015, 3, 11940-11947.

10 Z. Liu, K. Liu, F. Zhang, S. Jain, T. He, Y. Jiang, P. Liu, J. Yang, H. Liu and M. Yuan, Sol. Energy, 2020, 195, 436-445.

11 J. Yu, S. Badgujar, E. Jung, V. Singh, D. Kim, J. Gierschner, E. Lee, Y. Kim, S. Cho, M. Kwon and M. Song, Adv. Mater., 2019, 31, 1805554.

12 H. Pham, L. Gil-Escrig, K. Feron, S. Manzhos, S. Albrecht, H. Bolink and P. Sonar, J. Mater. Chem. A, 2019, 7, 12507-12517.

13 H. Huang, Y. Shih, L. Wang and K. Lin, Energy Environ. Sci., 2019, 12, 1265-1273.

14 C. Ng, K. Hamada, G. Kapil, M. Kamarudin, Z. Wang, S. Iikubo, Q. Shen, K. Yoshino, T. Minemoto and S. Hayase, J. Mater. Chem. A, 2020, 8, 2962-2968.

15 T. Yang, N. Jeon, H. Shin, S. Shin, Y. Kim and J. Seo, Adv. Sci., 2019, 6, 1900528.

16 F. Li, C. Zhang, J. Huang, H. Fan, H. Wang, P. Wang, C. Zhan, C. Liu, X. Li, L. Yang, Y. Song and K. Jiang, Angew. Chem., Int. Ed., 2019, 58, 6688-6692.

17 X. Shi, Y. Ding, S. Zhou, B. Zhang, M. Cai, J. Yao, L. Hu, J. Wu, S. Dai and M. Nazeeruddin, Adv. Sci., 2019, 6, 1901213.

18 X. Ren, Z. Wang and W. Choy, Adv. Opt. Mater., 2019, 7, 1900407.

19 P. Lopez-Varo, J. Jiménez-Tejada, M. García-Rosell, S. Ravishankar, G. Garcia-Belmonte, J. Bisquert and O. Almora, Adv. Energy Mater., 2018, 8, 1702772.

20 A. Kojima, K. Teshima, Y. Shirai and T. Miyasaka, J. Am. Chem. Soc., 2009, 131, 6050-6051.

21 J. Im, C. Lee, J. Lee, S. Park and N. Park, Nanoscale, 2011, 3, 4088-4093.

22 M. Liu, M. Johnston and H. Snaith, Nature, 2013, 501, 395.

23 H. Kim, C. Lee, J. Im, K. Lee, T. Moehl, A. Marchioro, S. Moon, R. Humphry-Baker, J. Yum, J. Moser, M. Grätzel and N. Park, Sci. Rep., 2012, 2, 591.

24 M. Lee, J. Teuscher, T. Miyasaka, T. Murakami and H. Snaith, Science, 2012, 338, 643-647.

25 L. Calio, S. Kazim, M. Grätzel and S. Ahmad, Angew. Chem., Int. Ed., 2016, 55, 14522-14545.

26 J. Burschka, N. Pellet, S. Moon, R. Humphry-Baker, P. Gao, M. Nazeeruddin and M. Grätzel, Nature, 2013, 499, 316.

27 M. Xiao, F. Huang, W. Huang, Y. Dkhissi, Y. Zhu, J. Etheridge, A. Gray-Weale, U. Bach, Y. Cheng and L. Spiccia, Angew. Chem., Int. Ed., 2014, 53, 9898-9903.

28 N. Jeon, H. Na, E. Jung, T. Yang, Y. Lee, G. Kim, H. Shin, S. Seok, J. Lee and J. Seo, Nat. Energy, 2018, 3, 682.

29 N. Park and K. Zhu, Nat. Rev. Mater., 2020, 5, 333-350.

30 K. Yoshikawa, H. Kawasaki, W. Yoshida, T. Irie, K. Konishi, K. Nakano, T. Uto, D. Adachi, M. Kanematsu, H. Uzu and K. Yamamoto, Nat. Energy, 2017, 2, 17032.
31 S. Bhattacharya and S. John, Sci. Rep., 2019, 9, 12482.

32 M. Tavakoli, M. Saliba, P. Yadav, P. Holzhey, A. Hagfeldt, S. Zakeeruddin and M. Grätzel, Adv. Energy Mater., 2019, 9, 1802646.

33 H. Li, L. Tao, F. Huang, Q. Sun, X. Zhao, J. Han, Y. Shen and M. Wang, ACS Appl. Mater. Interfaces, 2017, 9, 38967-38976.

34 P. Su, L. Liu, H. Yao, T. Liu, B. Zhang, J. Wang, S. Feng, A. Runa, W. Fu and H. Yang, Org. Electron., 2020, 77, 105490.

35 X. Cao, L. Zhi, Y. Li, F. Fang, X. Cui, Y. Yao, L. Ci, K. Ding and J. Wei, J. Mater. Chem. C, 2017, 5, 7458-7464.

36 Y. Shao, Z. Xiao, C. Bi, Y. Yuan and J. Huang, Nat. Commun., 2014, 5, 5784.

37 E. Aktas, J. Jiménez-López, C. Rodríguez-Seco, R. Pudi, M. Ortuño, N. López and E. Palomares, ChemPhysChem, 2019, 20, 2702-2711.

38 Y. Wu, A. Islam, X. Yang, C. Qin, J. Liu, K. Zhang, W. Peng and L. Han, Energy Environ. Sci., 2014, 7, 2934-2938.

39 A. Wakamiya, M. Endo, T. Sasamori, N. Tokitoh, Y. Ogomi, S. Hayase and Y. Murata, Chem. Lett., 2014, 43, 711-713.

40 Y. Jo, K. Oh, M. Kim, K. Kim, H. Lee, C. Lee and D. Kim, Adv. Mater. Interfaces, 2016, 3, 1500768.

41 N. Jeon, J. Noh, Y. Kim, W. Yang, S. Ryu and S. Seok, Nat. Mater., 2014, 13, 897.

42 K. Bush, K. Frohna, R. Prasanna, R. Beal, T. Leijtens, S. Swifter and M. McGehee, ACS Energy Lett., 2018, 3, 428-435.

43 C. Zuo, H. Bolink, H. Han, J. Huang, D. Cahen and L. Ding, Adv. Sci., 2016, 3, 1500324.

44 N. Jeon, J. Noh, W. Yang, Y. Kim, S. Ryu, J. Seo and S. Seok, Nature, 2015, 517, 476.

45 X. Shai, J. Wang, P. Sun, W. Huang, P. Liao, F. Cheng, B. Zhu, S. Chang, E. Yao, Y. Shen, L. Miao, Y. Yang and M. Wang, Nano Energy, 2018, 48, 117-127.

46 D. Zheng, G. Wang, W. Huang, B. Wang, W. Ke, J. Logsdon, H. Wang, Z. Wang, W. Zhu, J. Yu, M. Wasielewski, M. Kanatzidis, T. Marks and A. Facchetti, Adv. Funct. Mater., 2019, 29, 1900265.

47 Z. Wu, Z. Liu, Z. Hu, Z. Hawash, L. Qiu, Y. Jiang, L. Ono and Y. Qi, Adv. Mater., 2019, 31, 1804284.

48 Z. Liang, Z. Bi, K. Gao, Y. Fu, P. Guan, X. Feng, Z. Chai, G. Xu and X. Xu, Appl. Surf. Sci., 2019, 463, 939-946.

49 H. Pham, L. Gil-Escrig, K. Feron, S. Manzhos, S. Albrecht, H. Bolink and P. Sonar, J. Mater. Chem. A, 2019, 7, 12507-12517.

50 M. Kam, Q. Zhang, D. Zhang and Z. Fan, Sci. Rep., 2019, 9, 6963.

51 H. Sun, Y. Zhou, Y. Xin, K. Deng, L. Meng, J. Xiong and L. Li, Adv. Funct. Mater., 2019, 29, 1808667.

52 K. Huang, Y. Peng, Y. Gao, J. Shi, H. Li, X. Mo, H. Huang, Y. Gao, L. Ding and J. Yang, Adv. Energy Mater., 2019, 9, 1901419.

53 C. Liu, L. Zhang, X. Zhou, J. Gao, W. Chen, X. Wang and B. Xu, Adv. Funct. Mater., 2019, 29, 1807604.

54 H. Tan, A. Jain, O. Voznyy, X. Lan, F. Arquer, J. Fan, R. Quintero-Bermudez, M. Yuan, B. Zhang, Y. Zhao, 
F. Fan, P. Li, L. Quan, Y. Zhao, Z. Lu, Z. Yang, S. Hoogland and E. Sargent, Science, 2017, 355, 722-726.

55 J. Barbé, M. Tietze, M. Neophytou, B. Murali, E. Alarousu, A. Labban, M. Abulikemu, W. Yue, O. Mohammed, I. McCulloch, A. Amassian and S. Del Gobbo, ACS Appl. Mater. Interfaces, 2017, 9, 11828-11836.

56 P. Piotrowiak, Solar Energy Conversion: Dynamics of Interfacial Electron and Excitation Transfer, RSC Publishing, 2013.

57 A. Reshak, RSC Adv., 2014, 4, 39565-39571.

58 X. Zhao, S. Liu, H. Zhang, S. Chang, W. Huang, B. Zhu, Y. Shen, C. Shen, D. Wang, Y. Yang and M. Wang, Adv. Funct. Mater., 2019, 29, 1805168.

59 T. Liu, K. Chen, Q. Hu, R. Zhu and Q. Gong, Adv. Energy Mater., 2016, 6, 1600457.

60 Y. Zhang, X. Liu, P. Li, Y. Duan, X. Hu, F. Li and Y. Song, Nano Energy, 2019, 56, 733-740.

61 B. Tu, Y. Shao, W. Chen, Y. Wu, X. Li, Y. He, J. Li, F. Liu, Z. Zhang, Y. Lin, X. Lan, L. Xu, X. Shi, A. Ng, H. Li, L. Chung, A. Djurišić and Z. He, Adv. Mater., 2019, 31, 1805944.

62 K. Wojciechowski, M. Saliba, T. Leijtens, A. Abate and H. J. Snaith, Energy Environ. Sci., 2014, 7, 1142-1147.

63 G. Yang, H. Tao, P. Qin, W. Ke and G. Fang, J. Mater. Chem. A, 2016, 4, 3970-3990.

64 Q. Zhang, C. Dandeneau, X. Zhou and G. Cao, Adv. Mater., 2009, 21, 4087-4108.

65 Y. Yang, D. Ostrowski, R. France, K. Zhu, J. Lagemaat, J. Luther and M. Beard, Nat. Photonics, 2016, 10, 53.

66 H. Li, W. Shi, W. Huang, E. Yao, J. Han, Z. Chen, S. Liu, Y. Shen, M. Wang and Y. Yang, Nano Lett., 2017, 17, 2328-2335.

67 P. Jongh and D. Vanmaekelbergh, J. Phys. Chem. B, 1997, 101, 2716-2722.

68 K. Schwarzburg and F. Willig, Appl. Phys. Lett., 1991, 58, 2520-2522.

69 J. Bisquert, L. Bertoluzzi, I. Mora-Sero and G. GarciaBelmonte, J. Phys. Chem. C, 2014, 118, 18983-18991.

70 M. Wang, P. Chen, R. Humphry-Baker, S. Zakeeruddin and M. Grätzel, ChemPhysChem, 2009, 10, 290-299.

71 J. Bisquert, J. Phys. Chem. B, 2002, 106, 325-333.

72 M. Wang, C. Grätzel, S. Zakeeruddin and M. Grätzel, Energy Environ. Sci., 2012, 5, 9394-9405.

73 M. Wang, J. Liu, N. Cevey-Ha, S. Moon, P. Liska, R. HumphryBaker, J. Moser, C. Grätzel, P. Wang, S. Zakeeruddin and M. Grätzel, Nano Today, 2010, 5, 169-174.

74 H. Snaith and M. Grätzel, Adv. Mater., 2007, 19, 3643-3647. 75 W. Ke, G. Fang, J. Wang, P. Qin, H. Tao, H. Lei, Q. Liu, X. Dai and X. Zhao, ACS Appl. Mater. Interfaces, 2014, 6, 15959-15965.

76 W. Huang, E. Gann, N. Chandrasekaran, S. Prasad, S. Chang, L. Thomsen, D. Kabra, J. M. Hodgkiss, Y. Cheng and Y. Yang, Adv. Energy Mater., 2017, 7, 1602197.

77 W. Huang, E. Gann, N. Chandrasekaran, L. Thomsen, S. Prasad, J. Hodgkiss, D. Kabra, Y. Cheng and C. McNeill, Energy Environ. Sci., 2017, 10, 1843-1853.

78 M. Green, A. Ho-Baillie and H. Snaith, Nat. Photonics, 2014, 8, 506.
79 A. Marchioro, J. Teuscher, D. Friedrich, M. Kunst, R. Krol, T. Moehl, M. Grätzel and J. Moser, Nat. Photonics, 2014, 8, 250.

80 P. Chandrasekhar, A. Dubey, K. Reza, M. Hasan, B. Bahrami, V. Komarala, J. Hoefelmeyer, Q. He, F. Wu, H. Qiao, W. Zhang and Q. Qiao, Sustainable Energy Fuels, 2018, 2, 2260-2267.

81 A. West, I. Brodsky, C. Rahner, D. Woo, H. ErdjumentBromage, P. Tempst, M. Walsh, Y. Choi, G. Shadel and S. Ghosh, Nature, 2011, 472, 476.

82 G. Eperon, S. Stranks, C. Menelaou, M. Johnston, L. Herz and H. Snaith, Energy Environ. Sci., 2014, 7, 982-988.

83 S. Liu, W. Huang, P. Liao, N. Pootrakulchote, H. Li, J. Lu, J. Li, F. Huang, X. Shai, X. Zhao, Y. Shen, Y. Cheng and M. Wang, J. Mater. Chem. A, 2017, 5, 22952-22958.

84 V. Roiati, S. Colella, G. Lerario, L. Marco, A. Rizzo, A. Listorti and G. Gigli, Energy Environ. Sci., 2014, 7, 1889-1894.

85 R. Sanchez, V. Gonzalez-Pedro, J. Lee, N. Park, Y. Kang, I. Mora-Sero and J. Bisquert, J. Phys. Chem. Lett., 2014, 5, 2357-2363.

86 H. Snaith and M. Grätzel, Phys. Rev. Lett., 2007, 98, 177402.

87 L. Ming, H. Yang, W. Zhang, X. Zeng, D. Xiong, Z. Xu, H. Wang, W. Chen, X. Xu, M. Wang, J. Duan, Y. Cheng, J. Zhang, Q. Bao, Z. Wei and S. Yang, J. Mater. Chem. A, 2014, 2, 4566-4573.

88 P. Liao, X. Zhao, G. Li, Y. Shen and M. Wang, Nano-Micro Lett., 2018, 10, 5.

89 M. Wang, W. Yim, P. Liao and Y. Shen, ChemistrySelect, 2017, 2, 4469-4477.

90 D. Bi, W. Tress, M. Dar, P. Gao, J. Luo, C. Renevier, K. Schenk, A. Abate, F. Giordano, J. Baena, J. Decoppet, S. Zakeeruddin, M. Nazeeruddin, M. Grätzel and A. Hagfeldt, Sci. Adv., 2016, 2, e1501170.

91 W. Geng, C. Tong, J. Liu, W. Zhu, W. Lau and L. Liu, Sci. Rep., 2016, 6, 20131.

92 C. Zuo and L. Ding, Nanoscale, 2014, 6, 9935-9938.

93 W. Tress, Adv. Energy Mater., 2017, 7, 1602358.

94 L. Liu, A. Mei, T. Liu, P. Jiang, Y. Sheng, L. Zhang and H. Han, J. Am. Chem. Soc., 2015, 137, 1790-1793.

95 L. Zuo, Q. Chen, N. Marco, Y. Hsieh, H. Chen, P. Sun, S. Chang, H. Zhao, S. Dong and Y. Yang, Nano Lett., 2016, 17, 269-275.

96 M. Shahiduzzaman, S. Fukaya, E. Muslih, L. Wang, M. Nakano, M. Akhtaruzzaman, M. Karakawa, K. Takahashi, J.-M. Nunzi and T. Taima, Materials, 2020, 13, 2207.

97 J. Heo, M. You, M. Chang, W. Yin, T. Ahn, S. Lee, S. Sung, D. Kim and S. Im, Nano Energy, 2015, 15, 530-539.

98 Q. Liu, M. Qin, W. Ke, X. Zheng, Z. Chen, P. Qin, L. Xiong, H. Lei, J. Wan and J. Wen, Adv. Funct. Mater., 2016, 26, 6069-6075.

99 P. Tiwana, P. Docampo, M. Johnston, H. Snaith and L. Herz, ACS Nano, 2011, 5, 5158-5166.

100 D. Yang, R. Yang, K. Wang, C. Wu, X. Zhu, J. Feng, X. Ren, G. Fang, S. Priya and S. Liu, Nat. Commun., 2018, 9, 3239.

101 A. Yun, J. Kim, T. Hwang and B. Park, ACS Appl. Energy Mater., 2019, 2, 3554-3560. 
102 S. Shin, S. Lee and S. Seok, Adv. Funct. Mater., 2019, 29, 1900455.

103 J. Duan, Q. Yue, Q. Xiong, L. Wang, L. Zhu, K. Zhang, J. Zhang and H. Wang, Appl. Surf. Sci., 2019, 470, 613-621.

104 J. Lee, D. Shin, R. Rhee, S. Yun, K. Yeom, D. Chun, S. Lee, D. Kim, Y. Yi, J. Noh and J. Park, J. Phys. Chem. Lett., 2019, 10, 6545-6550.

105 Y. Zhou, X. Li and H. Lin, Small, 2020, 16, 1902579.

106 M. Caglar, Y. Caglar, S. Aksoy and S. Ilican, Appl. Surf. Sci., 2010, 256, 4966-4971.

107 P. Chandrasekhar, A. Dubey and Q. Qiao, Sol. Energy, 2020, 197, 78-83.

108 Z. Wang, J. Lou, X. Zheng, W. Zhang and Y. Qin, ACS Sustainable Chem. Eng., 2019, 7, 7421-7429.

109 X. Ling, J. Yuan, D. Liu, Y. Wang, Y. Zhang, Si Chen, H. Wu, F. Jin, F. Wu, G. Shi, Xun Tang, J. Zheng, S. Liu, Z. Liu and W. Ma, ACS Appl. Mater. Interfaces, 2017, 9, 23181-23188.

110 D. Shen, W. Zhang, Y. Li, A. Abate and M. Wei, ACS Appl. Nano Mater., 2018, 1, 4101-4109.

111 J. Feng, Z. Yang, D. Yang, X. Ren, X. Zhu, Z. Jin, W. Zi, Q. Wei and S. Liu, Nano Energy, 2017, 36, 1-8.

112 F. Ali, N. Pham, L. Fan, V. Tiong, K. Ostrikov, J. Bell, H. Wang and T. Tesfamichael, ACS Appl. Energy Mater., 2019, 2, 5456-5464.

113 Y. You, W. Tian, L. Min, F. Cao, K. Deng and L. Li, Adv. Mater. Interfaces, 2020, 7, 1901406.

114 S. Yoon, S. Kim, H. Kim, J. Park, I. Han, J. Jung and M. Park, Nanoscale, 2017, 9, 16305-16312.

115 W. Huang, B. Zhu, S. Chang, S. Zhu, P. Cheng, Y. Hsieh, L. Meng, R. Wang, C. Wang, C. Zhu, C. McNeill, M. Wang and Y. Yang, Nano Lett., 2018, 18, 5805-5811.

116 K. Jung, J. Lee, C. Im, J. Do, J. Kim, W. Chae and M. Lee, ACS Energy Lett., 2018, 3, 2410-2417.

117 S. Shin, W. Yang, J. Noh, J. Suk, N. Jeon, J. Park, J. Kim, W. Seong and S. Seok, Nat. Commun., 2015, 6, 7410.

118 C. Myung, G. Lee and K. Kim, J. Mater. Chem. A, 2018, 6, 23071-23077.

119 S. Shin, E. Yeom, W. Yang, S. Hur, M. Kim, J. Im, J. Seo, J. Noh and S. Seok, Science, 2017, 356, 167-171.

120 J. Fujisawa, T. Eda and M. Hanaya, Chem. Phys. Lett., 2017, 685, 23-26.

121 T. Mahmoudi, Y. Wang and Y. Hahn, Adv. Energy Mater., 2020, 10, 1903369.

122 H. Yu, H. Lu, F. Xie, S. Zhou and N. Zhao, Adv. Funct. Mater., 2016, 26, 1411-1419.

123 Y. Zhou, Z. Zhou, M. Chen, Y. Zong, J. Huang, S. Pang and N. Padture, J. Mater. Chem. A, 2016, 4, 17623-17635.

124 L. Xiong, M. Qin, G. Yang, Y. Guo, H. Lei, Q. Liu, W. Ke, H. Tao, P. Qin, S. Li, H. Yu and G. Fang, J. Mater. Chem. A, 2016, 4, 8374-8383.

125 H. Chen, D. Liu, Y. Wang, C. Wang, T. Zhang, P. Zhang, H. Sarvari, Z. Chen and S. Li, Nanoscale Res. Lett., 2017, 12, 238.

126 X. Ren, D. Yang, Z. Yang, J. Feng, X. Zhu, J. Niu, Y. Liu, W. Zhao and S. Liu, ACS Appl. Mater. Interfaces, 2017, 9, 2421-2429.

127 J. Fan, E. Menéndez, M. Guerrero, A. Quintana, E. Weschke, E. Pellicer and J. Sort, Nanomaterials, 2017, 7, 348.
128 D. Yang, R. Yang, X. Ren, X. Zhu, Z. Yang, C. Li and S. Liu, Adv. Mater., 2016, 28, 5206-5213.

129 D. Yang, X. Zhou, R. Yang, Z. Yang, W. Yu, X. Wang, C. Li, S. Liu and R. Chang, Energy Environ. Sci., 2016, 9, 3071-3078.

130 P. Gu, N. Wang, C. Wang, Y. Zhou, G. Long, M. Tian, W. Chen, X. Sun, M. Kanatzidis and Q. Zhang, J. Mater. Chem. A, 2017, 5, 7339-7344.

131 K. Jiang, F. Wu, H. Yu, Y. Yao, G. Zhang, L. Zhu and H. Yan, J. Mater. Chem. A, 2018, 6, 16868-16873.

132 H. Zhang, L. Xue, J. Han, Y. Fu, Y. Shen, Z. Zhang, Y. Li and M. Wang, J. Mater. Chem. A, 2016, 4, 8724-8733.

133 Y. Deng, S. Li, X. Li, R. Wang and X. Li, Electrochim. Acta, 2019, 326, 134924.

134 J. Wang, K. Datta, C. Weijtens, M. Wienk and R. Janssen, Adv. Funct. Mater., 2019, 29, 1905883.

135 K. Yao, S. Leng, Z. Liu, L. Fei, Y. Chen, S. Li, N. Zhou, J. Zhang, Y. Xu, L. Zhou, H. Huang and A. Jen, Joule, 2019, 3, 417-431.

136 W. Ke, C. Xiao, C. Wang, B. Saparov, H. Duan, D. Zhao, Z. Xiao, P. Schulz, S. Harvey, W. Liao, W. Meng, Y. Yu, A. Cimaroli, C. Jiang, K. Zhu, M. Al-Jassim, G. Fang, D. Mitzi and Y. Yan, Adv. Mater., 2016, 28, 5214-5221.

137 S. Shin, W. Yang, E. Yeom, S. Lee, N. Jeon, Y. Joo, I. Park, J. Noh and S. Seok, J. Phys. Chem. Lett., 2016, 7, 1845-1851.

138 D. Yang, R. Yang, J. Zhang, Z. Yang, S. Liu and C. Li, Energy Environ. Sci., 2015, 8, 3208-3214.

139 J. Xie, K. Huang, X. Yu, Z. Yang, K. Xiao, Y. Qiang, X. Zhu, L. Xu, P. Wang, C. Cui and D. Yang, ACS Nano, 2017, 11, 9176-9182.

140 Y. Zhou, S. Yang, X. Yin, J. Han, M. Tai, X. Zhao, H. Chen, Y. Gu, N. Wang and H. Lin, J. Mater. Chem. A, 2019, 7, 1878-1888.

141 X. Zhao, L. Tao, H. Li, W. Huang, P. Sun, J. Liu, S. Liu, Q. Sun, Z. Cui, L. Sun, Y. Shen, Y. Yang and M. Wang, Nano Lett., 2018, 18, 2442-2449.

142 X. Gong, Q. Sun, S. Liu, P. Liao, Y. Shen, C. Grätzel, S. Zakeeruddin, M. Grätzel and M. Wang, Nano Lett., 2018, 18, 3969-3977.

143 D. Li, C. Sun, H. Li, H. Shi, X. Shai, Q. Sun, J. Han, Y. Shen, H. Yip, F. Huang and M. Wang, Chem. Sci., 2017, 8, 4587-4594.

144 D. Yang, R. Yang, S. Priya and S. Liu, Angew. Chem., Int. Ed., 2019, 58, 4466-4483.

145 F. Huang, M. Li, P. Siffalovic, G. Cao and J. Tian, Energy Environ. Sci., 2019, 12, 518-549.

146 V. Corre, M. Stolterfoht, L. Toro, M. Feuerstein, C. Wolff, L. Gil-Escrig, H. Bolink, D. Neher and L. Koster, ACS Appl. Energy Mater., 2019, 2, 6280-6287.

147 M. Daboczi, I. Hamilton, S. Xu, J. Luke, S. Limbu, J. Lee, M. McLachlan, K. Lee, J. Durrant, I. Baikie and J. Kim, ACS Appl. Mater. Interfaces, 2019, 11, 46808-46817.

148 W. Chang, H. Tian, G. Fang, D. Guo, Z. Wang and K. Zhao, Sol. Energy, 2019, 186, 323-327.

149 D. Pyscha, A. Mettea and S. Glunz, Sol. Energy Mater. Sol. Cells, 2007, 91, 1698-1706.

$150 \mathrm{~K}$. Young, Advances in Optical and Photonic Devices\| Resonant Tunnelling Optoelectronic Circuits, 2010, ch. 10, DOI: $10.5772 / 127$. 